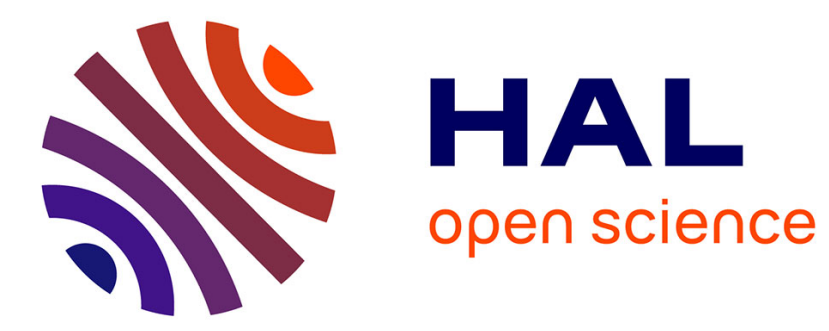

\title{
Finite-temperature coarse-graining of one-dimensional models: mathematical analysis and computational approaches
}

\author{
Xavier Blanc, Claude Le Bris, Frédéric Legoll, Carsten Patz
}

\section{To cite this version:}

Xavier Blanc, Claude Le Bris, Frédéric Legoll, Carsten Patz. Finite-temperature coarse-graining of one-dimensional models: mathematical analysis and computational approaches. Journal of Nonlinear Science, 2010, 20 (2), pp.241-275. 10.1007/s00332-009-9057-y . inria-00282107v2

\section{HAL Id: inria-00282107 https://hal.inria.fr/inria-00282107v2}

Submitted on 28 May 2008

HAL is a multi-disciplinary open access archive for the deposit and dissemination of scientific research documents, whether they are published or not. The documents may come from teaching and research institutions in France or abroad, or from public or private research centers.
L'archive ouverte pluridisciplinaire HAL, est destinée au dépôt et à la diffusion de documents scientifiques de niveau recherche, publiés ou non, émanant des établissements d'enseignement et de recherche français ou étrangers, des laboratoires publics ou privés. 


\section{N R I A}

INSTITUT NATIONAL DE RECHERCHE EN INFORMATIQUE ET EN AUTOMATIQUE

Finite-temperature coarse-graining of one-dimensional models: mathematical analysis and computational approaches

Xavier Blanc — Claude Le Bris — Frédéric Legoll — Carsten Patz

$\mathrm{N}^{\circ} 6544$

May 2008

Thème NUM 



\title{
RIN RIA
}

\section{Finite-temperature coarse-graining of one-dimensional models: mathematical analysis and computational approaches}

\author{
Xavier Blanc $^{* \dagger}$, Claude Le Bris ${ }^{\ddagger \dagger}$, Frédéric Legoll ${ }^{\S \dagger}$, Carsten Patz \\ Thème NUM — Systèmes numériques \\ Projet MICMAC
}

Rapport de recherche $n^{\circ} 6544$ - May 2008 - 40 pages

\begin{abstract}
We present a possible approach for the computation of free energies and ensemble averages of one-dimensional coarse-grained models in materials science. The approach is based upon a thermodynamic limit process, and makes use of ergodic theorems and large deviations theory. In addition to providing a possible efficient computational strategy for ensemble averages, the approach allows for assessing the accuracy of approximations commonly used in practice.
\end{abstract}

Key-words: ensemble averages, free energies, coarse-grained models, materials science, thermodynamic limit, computational strategy

* Laboratoire J.-L. Lions, Université Pierre et Marie Curie, Boîte courrier 187, 75252 Paris Cedex 05, France. Contact: blanc@ann.jussieu.fr

$\dagger$ INRIA Rocquencourt, MICMAC team-project, Domaine de Voluceau, B.P. 105, 78153 Le Chesnay Cedex, France.

$\ddagger$ CERMICS, Ecole Nationale des Ponts et Chaussées, 6 et 8 avenue Blaise Pascal, Cité Descartes, 77455 Marne-la-Vallée Cedex 2, France. Contact: lebris@cermics.enpc.fr

$\S$ LAMI, Ecole Nationale des Ponts et Chaussées, 6 et 8 avenue Blaise Pascal, Cité Descartes, 77455 Marne-la-Vallée Cedex 2, France. Contact: legoll@lami.enpc.fr

T Weierstrass-Institut für Angewandte Analysis und Stochastik, Mohrenstrasse 39, 10117 Berlin, Germany. Contact: patz@wias-berlin.de 


\section{Modèles réduits pour les systèmes atomistiques mono-dimensionnels à température finie: analyse mathématique et approches numériques}

Résumé : Nous présentons une approche possible pour le calcul de moyennes d'ensemble et d'énergies libres de modèles réduits en science des matériaux, dans un cadre monodimensionnel. L'approche s'appuie sur une limite thermodynamique, et utilise des théorèmes ergodiques et la théorie des grandes déviations. Nous obtenons ainsi non seulement une stratégie numérique efficace pour le calcul de moyennes thermodynamiques, mais aussi un moyen de vérifier numériquement la validité des hypothèses qui sont couramment faites dans la littérature pour développer de telles approches.

Mots-clés : moyennes d'ensemble, énergies libres, modèles réduits, science des matériaux, limite thermodynamique, stratégie numérique 


\section{Introduction}

Computing canonical averages is a standard task of computational materials science. Consider an atomistic, supposedly large, system consisting of $N$ particles, at positions $u=$ $\left(u^{1}, \ldots, u^{N}\right) \in \mathbb{R}^{3 N}$. Provide this system with an energy

$$
E_{\mu}(u)=E_{\mu}\left(u^{1}, \ldots, u^{N}\right)
$$

A prototypical example of such an energy is the pair interaction energy

$$
E_{\mu}(u)=\frac{1}{2} \sum_{i \neq j} W\left(u^{j}-u^{i}\right) .
$$

The finite temperature thermodynamical properties of the material are obtained from canonical ensemble averages,

$$
\langle A\rangle=\frac{\int_{\Omega^{N}} A(u) \exp \left(-\beta E_{\mu}(u)\right) d u}{\int_{\Omega^{N}} \exp \left(-\beta E_{\mu}(u)\right) d u},
$$

where $\Omega \subset \mathbb{R}^{3}$ is the macroscopic domain where the positions $u^{i}$ vary, $A$ is the observable of interest, and $\beta=1 /\left(k_{B} T\right)$ is the inverse temperature [15]. The denominator of (3) is denoted by $Z$ and called the partition function. The major computational difficulty in (3) is of course the $N$-fold integrals, where $N$, the number of particles, is extremely large. For integrals of the type (3) to be quantitatively meaningful in practice, $N$ does not need to approach the Avogadro number, but still needs to be extremely large $\left(10^{5}\right.$, say).

The three dominant computational approaches for the evaluation of (3) are Monte Carlo methods, Markov chains methods, and Molecular dynamics methods respectively (see e.g. [13] for a review and a mathematical and computational comparison). In the present article, we use the latter type of methods, and more precisely the overdamped Langevin dynamics (also called biased randow walk). The ensemble average (3) is calculated as the long-time average

$$
\langle A\rangle=\lim _{T \rightarrow+\infty} \frac{1}{T} \int_{0}^{T} A(u(t)) d t
$$

along the trajectory generated by the stochastic differential equation

$$
d u=-\nabla_{u} E_{\mu}(u) d t+\sqrt{2 / \beta} d B_{t} .
$$

It is often the case that the observable $A$ actually does not depend on the positions $u^{i}$ of all the atoms, but only on some of them. Think for instance of nanoindentation: we are especially interested in the positions of the atoms below the indenter, in the forces applied on these atoms, ... Our first aim is to design a numerical method that efficiently computes canonical averages of such observables. Our second aim will be to assess the validity of other approaches, as compared to ours. 
The QuasiContinuum Method (QCM) is a commonly used example of approaches that allow for the calculation of ensemble averages. In its original version, the method was focused on the zero temperature setting. It was originally introduced in [44, 45], and then further developed in $[27,34,35,41,42,43]$. It has been studied mathematically in e.g. $[1,2,3,7,8,9,18,20,21,22,30,31,37]$. See [10] for a recent review. An extension of the original idea has recently been developed in [19] and carries through to the finite-temperature case, considered in the present article. See also [14, 29] for prior studies developing ideas in the same vein.

Let us briefly detail the bottom line of coarse-graining strategies for the computation of canonical averages. For simplicity of exposition, we let the atoms vary in $\Omega=\mathbb{R}^{3}$. The idea is to subdivide the particles of the system into two subsets. The first subset consists of the so-called representative atoms (abbreviated in the QCM terminology as repatoms, with positions henceforth denoted by $u_{r}$ ). The second subset is that of atoms that are eliminated in the coarse-grained procedure. Their positions are denoted here by $u_{c}$. We assume that the observable considered only depends on the positions $u_{r}$ of the repatoms, not on those of the other atoms, $u_{c}$. More precisely, one writes

$$
u=\left(u^{1}, \ldots, u^{N}\right)=\left(u_{r}, u_{c}\right), \quad u_{r} \in \mathbb{R}^{3 N_{r}}, \quad u_{c} \in \mathbb{R}^{3 N_{c}}, \quad N=N_{r}+N_{c} .
$$

Our aim is to compute (3) for such observable, that is,

$$
\langle A\rangle=Z^{-1} \int_{\mathbb{R}^{3 N}} A\left(u_{r}\right) \exp \left(-\beta E_{\mu}(u)\right) d u .
$$

We observe that, owing to our assumption on $A$,

$$
\int_{\mathbb{R}^{3 N}} A\left(u_{r}\right) \exp \left(-\beta E_{\mu}(u)\right) d u=\int_{\mathbb{R}^{3 N_{r}}} d u_{r} A\left(u_{r}\right) \int_{\mathbb{R}^{3 N_{c}}} \exp \left(-\beta E_{\mu}\left(u_{r}, u_{c}\right)\right) d u_{c}
$$

and likewise

$$
Z=\int_{\mathbb{R}^{3 N}} \exp \left(-\beta E_{\mu}(u)\right) d u=\int_{\mathbb{R}^{3 N_{r}}} d u_{r} \int_{\mathbb{R}^{3 N_{c}}} \exp \left(-\beta E_{\mu}\left(u_{r}, u_{c}\right)\right) d u_{c} .
$$

Introducing the coarse-grained potential (also called free energy)

$$
E_{\mathrm{CG}}\left(u_{r}\right):=-\frac{1}{\beta} \ln \left[\int_{\mathbb{R}^{3 N_{c}}} \exp \left(-\beta E_{\mu}\left(u_{r}, u_{c}\right)\right) d u_{c}\right],
$$

the expression (6) rewrites

$$
\langle A\rangle=Z_{r}^{-1} \int_{\mathbb{R}^{3 N_{r}}} A\left(u_{r}\right) \exp \left(-\beta E_{\mathrm{CG}}\left(u_{r}\right)\right) d u_{r},
$$

where of course

$$
Z_{r}=\int_{\mathbb{R}^{3 N_{r}}} \exp \left(-\beta E_{\mathrm{CG}}\left(u_{r}\right)\right) d u_{r}
$$


Under appropriate conditions ensuring ergodicity of the system, the integral (8) is in turn computed from

$$
\langle A\rangle=\lim _{T \rightarrow+\infty} \frac{1}{T} \int_{0}^{T} A\left(u_{r}(t)\right) d t \text { with } d u_{r}=-\nabla_{u_{r}} E_{\mathrm{CG}}\left(u_{r}\right) d t+\sqrt{2 / \beta} d B_{t} .
$$

Simulating the dynamics in (10) is a less computationally demanding task than simulating (5), owing to the reduced dimension $N_{r}$. This simplification comes at a price: calculating the coarse-grained free energy (7).

Remark 1 The present work concentrates on the computation of ensemble averages using coarse-grained models, and free energies. Practice shows that the same coarse-graining paradigm is used to simulate actual coarse-grained dynamics at finite temperature. We will not go in this direction as the physical relevance of the latter approach is unclear to us.

In order to approximate the free energy (7), state-of-the-art finite temperature methods perform a Taylor expansion of the position of the eliminated atoms $u_{c}$. In this Taylor expansion, a linear interpolation and a harmonic approximation of the positions of the atoms are successively performed. More precisely, given the positions $u_{r}$ of the repatoms, the average positions $\overline{u_{c}}\left(u_{r}\right)$ of the eliminated atoms are first determined by linear interpolation between two (or more) adjacent repatoms. Then it is postulated that

$$
u_{c}=\overline{u_{c}}\left(u_{r}\right)+\xi_{c}
$$

where the perturbation $\xi_{c}$ is small. The energy is then calculated from a Taylor expansion truncated at second order:

$$
\begin{aligned}
E_{\mu}\left(u_{r}, u_{c}\right) & =E_{\mu}\left(u_{r}, \overline{u_{c}}\left(u_{r}\right)+\xi_{c}\right) \\
& \approx E_{\mu}\left(u_{r}, \overline{u_{c}}\left(u_{r}\right)\right)+\frac{\partial E_{\mu}}{\partial u_{c}}\left(u_{r}, \overline{u_{c}}\left(u_{r}\right)\right) \cdot \xi_{c}+\frac{1}{2} \xi_{c} \cdot \frac{\partial^{2} E_{\mu}}{\partial u_{c}^{2}} \cdot \xi_{c}=: \widetilde{E}\left(u_{r}, u_{c}\right) .
\end{aligned}
$$

It follows (we skip the details of the argument and refer to the bibliography pointed out above for further details) that $E_{\mathrm{CG}}\left(u_{r}\right)$ is approximated by

$$
E_{\mathrm{QCM}}\left(u_{r}\right)=-\frac{1}{\beta} \ln \int_{\mathbb{R}^{3 N_{c}}} \exp \left(-\beta \widetilde{E}\left(u_{r}, u_{c}\right)\right) d u_{c},
$$

which is analytically computable. Without such simplifying assumptions, the actual computation of $E_{\mathrm{CG}}$ for practical values of $N_{r}$ and $N_{c}$ seems undoable. The approach has proven efficient. Reportedly, it satisfactorily treats three-dimensional problems of large size. However, from the mathematical standpoint, it is an open question to evaluate the impact of the above couple of approximations (linear interpolation of the average positions, followed by harmonic expansion). The purpose of the present article is to present an approach that, in simple cases and under specific assumptions, also allows for a quantitative assessment of the validity and limits of the above couple of approximations. 
Our approach is based on a thermodynamic limit. It was first outlined in [38] for the special case of harmonic interactions. The approach is exact in the limit of an infinite number of eliminated atoms, and therefore valid when this number $N_{c}$ is large as compared to the number $N_{r}$ of representative atoms that are kept explicit in the coarse-grained model. This regime, after all, is the regime that all effective coarse-graining strategies should target, although, in practice, $N_{c}$ is seemingly much smaller than ideally, and even sometimes of the same order of magnitude as $N_{r}$. In short, the consideration of the asymptotic limit $N_{c} \rightarrow+\infty$ makes tractable a computation which is not tractable for finite $N_{c}$ (unless simplifications, as those mentioned above, are performed). We do not claim for originality in our theoretical considerations on the thermodynamic limit of the free energy of atomistic systems. We provide them here for consistency. However, our specific use of such theoretical considerations as a computational strategy for approximating coarse-grained ensemble averages in computational materials science seems, to the best of our knowledge, new. We were not able to find any comparable endeavour in the existing literature we have access to.

Let us conclude this introduction by briefly describing our approach. Assume for simplicity that there is only one repatom: $N_{r}=1$. Our idea to compute $\langle A\rangle$ in (6) is to change variables, that is introduce $y=\left(y_{1}, \ldots, y_{N}\right)=\Phi(u)$, and recast (6) as

$$
\langle A\rangle=\int_{\mathbb{R}^{3 N}} A\left(\frac{1}{N} \sum_{i=1}^{N} y_{i}\right) \nu(y) d y
$$

for some probability density $\nu(y)$ (see equation (17) below for an explicit example). We next recognize $\langle A\rangle$ as the expectation value $\mathbb{E}\left[A\left(\frac{1}{N} \sum_{i=1}^{N} Y_{i}\right)\right]$, where $Y=\left(Y_{1}, \ldots, Y_{N}\right)$ are random variables distributed according to the probability $\nu$. A Law of Large Numbers provides the limit of $\langle A\rangle$ when $N \rightarrow+\infty$ (which corresponds to $N_{c} \rightarrow+\infty$, since $N_{r}=1$ ). The rate of convergence may also be evaluated using the Central Limit Theorem.

The above approach bypasses the calculation of the free energy $E_{\mathrm{CG}}$ to compute the ensemble average (8). But it is also interesting to try and evaluate $E_{\mathrm{CG}}$ in the same regime, in order to, again, both provide an efficient numerical approach and assess the validity of commonly used simplifying approaches. First, it is to be remarked that $E_{\mathrm{CG}}$ scales linearly with the number $N_{c}$ of eliminated atoms. The relevant quantity is hence the free energy per particle

$$
F_{\infty}\left(u_{r}\right):=\lim _{N_{c} \rightarrow+\infty} \frac{1}{N_{c}} E_{\mathrm{CG}}\left(u_{r}\right) .
$$

This energy is related to the coarse-grained constitutive law of the material at finite temperature (see $[17,36]$ for related approaches). Even if $F_{\infty}$ is a good approximation of $E_{\mathrm{CG}} / N_{c}$ for large $N_{c}$, it can be seen that $N_{c} F_{\infty}$ is not necessarily a good approximation of $E_{\mathrm{CG}}$. It is not clear to us how to use the probability measure $Z_{N_{c}}^{-1} \exp \left(-\beta N_{c} F_{\infty}\right)$ to compute in an efficient manner an approximation of the average $\langle A\rangle$ (see Remark 6 below).

We develop our approach in the one-dimensional setting, for simple cases of pair interactions. We first consider nearest neighbour (NN) interactions. In this case, we develop a 
computational strategy to approximate ensemble averages (see Section 2.1), and we next address the computations of free energies (see Section 2.2). Numerical considerations are collected in Section 2.3.

We next turn to next-to-nearest neighbour interactions, traditionally abbreviated as NNN. For this model, we focus on the computation of ensemble averages (see Section 3.1). As explained in Section 3.2, more complicated types of interaction potentials and "essentially one-dimensional systems" (including polymer chains) may be treated likewise, although we do not pursue in this direction.

A similar interpretation of ensemble averages as the one presented here, using a Markov chain formalism, should lead to an analogous strategy for two-dimensional systems. Some preliminary developments, not included in the present article, already confirm this. However, definite conclusions are yet to be obtained, both on the formal validity of the approach and on the best possible numerical efficiency accomplished. The fact that the two-dimensional case is much more difficult than the one-dimensional case is corroborated by the literature on this subject: only very simple cases, such as spin systems, or harmonic interactions (with zero equilibrium length) are known to have explicit solutions in this context (see the reviews $[5,39])$. We therefore prefer to postpone considerations on the two-dimensional situation until a future publication [11].

\section{The nearest neighbour (NN) case}

As mentioned in the introduction, our approach is based on the asymptotic limit $N \longrightarrow+\infty$. We therefore first rescale the problem with the interatomic distance $h$, such that $N h=L=1$. The atomistic energy (2) in the rescaled NN case writes

$$
E_{\mu}\left(u^{1}, \ldots, u^{N}\right)=\sum_{i=1}^{N} W\left(\frac{u^{i}-u^{i-1}}{h}\right) .
$$

We now impose $u^{0}=0$ to avoid translation invariance, and consider that only atoms 0 and $N$ are repatoms, while all the other atoms $i=1, \ldots, N-1$ are eliminated in the coarsegraining procedure (see Figure 1). Our argument can be straightforwardly adapted to treat the case of $N_{r} \gg 2$ repatoms (see Figure 2). See the end of Section 2.1 for this.

$\mathrm{L}$

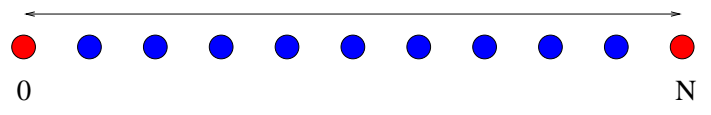

Figure 1: We isolate a segment between two consecutive repatoms (in red). All atoms in-between (in blue) are eliminated in the coarse-graining procedure. 
0

Figure 2: The repatoms (in red) are explicitly treated, all other atoms (in blue) being eliminated in the $\mathrm{CG}$ procedure.

In this simple situation, the average (6) reads

$$
\langle A\rangle_{N}=Z^{-1} \int_{\mathbb{R}^{N}} A\left(u^{N}\right) \exp \left(-\beta \sum_{i=1}^{N} W\left(\frac{u^{i}-u^{i-1}}{h}\right)\right) d u^{1} \ldots d u^{N},
$$

where we have explicitly mentioned the dependence of $\langle A\rangle$ upon $N$ using a subscript. We introduce

$$
y_{i}:=\frac{u^{i}-u^{i-1}}{h}, \quad i=1, \ldots, N
$$

and next remark that

$$
u^{N}=h \sum_{i=1}^{N} y_{i}=\frac{1}{N} \sum_{i=1}^{N} y_{i}
$$

The average rewrites as

$$
\langle A\rangle_{N}=Z^{-1} \int_{\mathbb{R}^{N}} A\left(\frac{1}{N} \sum_{i=1}^{N} y_{i}\right) \exp \left(-\beta \sum_{i=1}^{N} W\left(y_{i}\right)\right) d y^{1} \ldots d y^{N},
$$

where now $Z=\int_{\mathbb{R}^{N}} \exp \left(-\beta \sum_{i=1}^{N} W\left(y_{i}\right)\right) d y^{1} \ldots d y^{N}$.

Remark 2 In (14), we let the variables $u^{i}$ vary on the whole real line. We do not constrain them to obey $u^{i-1} \leq u^{i}$, which encodes the fact that nearest neighbours remain nearest neighbours. The argument provided here and below carries through when this constraint is accounted for, basically replacing the interaction potential $W$ by

$$
W_{c}(y)= \begin{cases}W(y) & \text { when } y \geq 0 \\ +\infty & \text { otherwise }\end{cases}
$$

Likewise, we could also impose that all the $u^{i}$ stay in a given macroscopic segment. If they are ordered increasingly, it suffices to impose this constraint on $u^{0}$ and $u^{N}$. This is again a simple modification of our argument. 


\subsection{Limit of the average}

It is evident on the expression (17) that

$$
\langle A\rangle_{N}=\mathbb{E}\left[A\left(\frac{1}{N} \sum_{i=1}^{N} Y_{i}\right)\right]
$$

for independent identically distributed (i.i.d.) random variables $Y_{i}$, sharing the common law $z^{-1} \exp (-\beta W(y)) d y$, with $z=\int_{\mathbb{R}} \exp (-\beta W(y)) d y$. A simple computation thus gives

Theorem 1 Assume that $A: \mathbb{R} \longrightarrow \mathbb{R}$ is continuous, that for some $p \geq 1$, there exists a constant $C>0$ such that

$$
\forall y \in \mathbb{R}, \quad|A(y)| \leq C\left(1+|y|^{p}\right)
$$

and that

$$
\int_{\mathbb{R}}\left(1+|y|^{p}\right) \exp (-\beta W(y)) d y<+\infty
$$

Then,

$$
\lim _{N \rightarrow+\infty}\langle A\rangle_{N}=A\left(y^{*}\right), \quad \text { where } \quad y^{*}:=z^{-1} \int_{\mathbb{R}} y \exp (-\beta W(y)) d y
$$

with $z=\int_{\mathbb{R}} \exp (-\beta W(y)) d y$. In addition, if $A$ is $C^{2}$ and if (19)-(20) hold with $p=2$, then

$$
\langle A\rangle_{N}=\langle A\rangle_{N}^{\text {approx }, 1}+o\left(\frac{1}{N}\right)
$$

with

$$
\langle A\rangle_{N}^{\text {approx }, 1}:=A\left(y^{*}\right)+\frac{\sigma^{2}}{2 N} A^{\prime \prime}\left(y^{*}\right), \quad \text { where } \quad \sigma^{2}=z^{-1} \int_{\mathbb{R}}\left(y-y^{*}\right)^{2} \exp (-\beta W(y)) d y .
$$

The proof of (21) is a direct application of the Law of Large Numbers, and that of (22) is an application of the Central Limit Theorem. We skip them. The following considerations, for more regular observables $A$, indeed contain the ingredients for proving (21)-(22), simply by truncating the expansion at first order.

Note that if $A$ is more regular than stated in Theorem 1, then it is of course possible to proceed further in the expansion of $\langle A\rangle_{N}$ in powers of $1 / N$. Indeed, assume for instance 
that $A$ is $C^{6}$, that $A^{(6)}$ is globally bounded and that (19)-(20) hold with $p=6$. Then

$$
\begin{aligned}
A\left(\frac{1}{N} \sum_{i=1}^{N} Y_{i}\right)= & A\left(y^{*}+\frac{1}{N} \sum_{i=1}^{N} D_{i}\right) \\
= & A\left(y^{*}\right)+A^{\prime}\left(y^{*}\right) \frac{1}{N} \sum_{i=1}^{N} D_{i}+\frac{1}{2} A^{\prime \prime}\left(y^{*}\right)\left(\frac{1}{N} \sum_{i=1}^{N} D_{i}\right)^{2} \\
& +\frac{1}{6} A^{(3)}\left(y^{*}\right)\left(\frac{1}{N} \sum_{i=1}^{N} D_{i}\right)^{3}+\frac{1}{24} A^{(4)}\left(y^{*}\right)\left(\frac{1}{N} \sum_{i=1}^{N} D_{i}\right)^{4} \\
& +\frac{1}{5 !} A^{(5)}\left(y^{*}\right)\left(\frac{1}{N} \sum_{i=1}^{N} D_{i}\right)^{5}+\frac{1}{6 !} A^{(6)}(\xi)\left(\frac{1}{N} \sum_{i=1}^{N} D_{i}\right)^{6},
\end{aligned}
$$

where $D_{i}=Y_{i}-y^{*}$ and $\xi$ lies between $y^{*}$ and $(1 / N) \sum Y_{i}$. We now take the expectation value of this equality. Let us introduce

$$
\begin{aligned}
\langle A\rangle_{N}^{\text {approx }, 2}:= & A\left(y^{*}\right)+\frac{1}{2} A^{\prime \prime}\left(y^{*}\right) \frac{1}{N} \mathbb{E}\left(D_{1}^{2}\right)+\frac{1}{6} A^{(3)}\left(y^{*}\right) \frac{1}{N^{2}} \mathbb{E}\left(D_{1}^{3}\right) \\
& +\frac{1}{24} A^{(4)}\left(y^{*}\right)\left(\frac{1}{N^{3}} \mathbb{E}\left(D_{1}^{4}\right)+\frac{N-1}{N^{3}}\left(\mathbb{E}\left(D_{1}^{2}\right)\right)^{2}\right) \\
& +\frac{1}{5 !} A^{(5)}\left(y^{*}\right)\left(\frac{1}{N^{4}} \mathbb{E}\left(D_{1}^{5}\right)+\frac{N-1}{N^{4}} \mathbb{E}\left(D_{1}^{2}\right) \mathbb{E}\left(D_{1}^{3}\right)\right)
\end{aligned}
$$

Then

$$
\left|\langle A\rangle_{N}-\langle A\rangle_{N}^{\text {approx }, 2}\right| \leq \frac{1}{6 !}\left\|A^{(6)}\right\|_{L^{\infty}} \mathbb{E}\left[\left(\frac{1}{N} \sum_{i=1}^{N} D_{i}\right)^{6}\right] .
$$

We now use the fact that any i.i.d. variables $D_{i}$ with mean value 0 satisfy the following bounds:

$$
\forall p \in \mathbb{N}, \quad \exists C_{p}>0, \quad\left|\mathbb{E}\left[\left(\frac{1}{N} \sum_{i=1}^{N} D_{i}\right)^{p}\right]\right| \leq \begin{cases}\frac{C_{p}}{N^{\frac{p}{2}}} & \text { if } p \text { is even; } \\ \frac{C_{p}}{N^{\frac{p+1}{2}}} & \text { if } p \text { is odd. }\end{cases}
$$

This is proved by developping the power $p$ of the sum, and then using the fact that the variables are i.i.d and have mean value zero. We hence infer from (24), (25) and (26) that

$$
\langle A\rangle_{N}=A\left(y^{*}\right)+\frac{\sigma^{2}}{2 N} A^{\prime \prime}\left(y^{*}\right)+\frac{1}{N^{2}}\left(\frac{a_{3}}{6} A^{(3)}\left(y^{*}\right)+\frac{\sigma^{4}}{24} A^{(4)}\left(y^{*}\right)\right)+O\left(\frac{1}{N^{3}}\right),
$$

where $\sigma$ is defined by (23) and

$$
a_{3}=z^{-1} \int_{\mathbb{R}}\left(y-y^{*}\right)^{3} \exp (-\beta W(y)) d y .
$$


More generally, it is possible to expand $\langle A\rangle_{N}$ at any order in $1 / N$, provided that $A$ is sufficiently smooth and $\exp (-\beta W)$ sufficiently small at infinity. In view of the bounds (26), we can see that using a Taylor expansion of order $2 p$ around $y^{*}$ for $A$ gives an expansion of $\langle A\rangle_{N}$ of order $p$.

The practical consequence of Theorem 1 is that, for computational purposes, we may take the approximation

$$
\langle A\rangle_{N} \approx A\left(z^{-1} \int_{\mathbb{R}} u^{N} \exp \left(-\beta W\left(u^{N}\right)\right) d u^{N}\right) .
$$

As pointed out above, it is possible to improve this approximation if necessary by expanding further in powers of $1 / N$.

We conclude this section by showing that our consideration of a single "segment" carries through to the case when there are 3 repatoms, of respective index $0, M_{1}$ and $M_{1}+M_{2}$, with $M_{1} h=L_{1}, M_{2} h=L_{2}, N h=L=1$ (see Figure 3 ). The average to compute writes

$$
\langle A\rangle_{N}=Z^{-1} \int_{\mathbb{R}^{N}} A\left(u^{M_{1}}, u^{M_{1}+M_{2}}\right) \exp \left(-\beta \sum_{i=1}^{N} W\left(\frac{u^{i}-u^{i-1}}{h}\right)\right) d u^{1} \ldots d u^{N} .
$$

In the regime $h \rightarrow 0, N, M_{1}, M_{2} \rightarrow+\infty$ with $M_{1} / N$ and $M_{2} / N$ fixed, we have, using similar arguments,

$$
\lim _{N \rightarrow+\infty}\langle A\rangle_{N}=A\left(L_{1} y^{*}, L_{2} y^{*}\right) .
$$

The generalization to $N_{r}>3$ repatoms, in the appropriate asymptotic regime, easily follows.

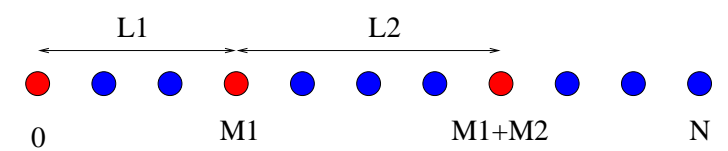

Figure 3: When considering two consecutive segments -or more-, the argument may be readily adapted. See the text.

Remark 3 (The small temperature limit) It is interesting here to consider the small temperature limit of the above expansion, that is, the limit $\beta \rightarrow+\infty$. In such a case, using the Laplace method (see [6]), it is possible to compute the limit of the various terms that appear in the expansion of $\langle A\rangle_{N}$ (see (27)). We give as an example the first and the second terms:

$$
A\left(y^{*}\right)=A(a)+O\left(\frac{1}{\beta}\right), \quad \frac{\sigma^{2}}{2} A^{\prime \prime}\left(y^{*}\right)=\frac{1}{\beta} \frac{A^{\prime \prime}(a)}{W^{\prime \prime}(a)}+O\left(\frac{1}{\beta^{2}}\right),
$$


where $a$ is the point where $W$ attains its minimum (in this remark, we assume for simplicity that $W$ attains its minimum at a unique point). It is possible to recover these terms by expanding the energy $E_{\mu}$ around the equilibrium configuration corresponding to $y_{i}=a$. Indeed, if we assume that $W(y)=W^{\prime \prime}(a)(y-a)^{2} / 2$ in (14), then a simple explicit computation gives

$$
\langle A\rangle_{N}=A(a)+\frac{1}{2 N \beta W^{\prime \prime}(a)} A^{\prime \prime}(a)+O\left(\frac{1}{\beta^{2} N^{2}}\right) .
$$

Hence, expanding the first terms of (23) in powers of $1 / \beta$ for large $\beta$ gives an expansion that agrees with that obtained using a harmonic approximation of the energy. This provides a quantitative evaluation of the latter approach in this asymptotic regime.

\subsection{Limit of the free energy}

We now look for a more demanding result. For clarity, let us return to the general coarsegrained average (8), which of course equals (14) and (17) in our simple NN case. Instead of searching for the limit of the average $\langle A\rangle$ for large $N_{c}$, we now look for the limit of the free energy per particle (see (7) and (12)).

In the present section, $u_{r}$ is in fact equal to $u^{N}$ (the right end atom) since $u^{0}=0$, although a repatom, is fixed to avoid translation invariance. Thus we wish to identify the behaviour for $N$ large of

$$
E_{\mathrm{CG}}\left(u^{N}\right)=-\frac{1}{\beta} \ln \left[\int_{\mathbb{R}^{N-1}} \exp \left(-\beta E_{\mu}\left(u^{1}, \ldots, u^{N}\right)\right) d u^{1} \ldots u^{N-1}\right] .
$$

Note that $E_{\mathrm{CG}}$ is the free energy corresponding to integrating out $N-1$ variables. From Thermodynamics, it is expected that $E_{\mathrm{CG}}$ scales linearly with $N$. This is confirmed by the consideration of an harmonic potential $W(x)=\frac{k}{2}(x-a)^{2}$, for which

$$
E_{\mathrm{CG}}\left(u^{N}\right)=\frac{k N}{2}\left(u^{N}-a\right)^{2}+C(N, \beta, k)
$$

where $C(N, \beta, k)=\frac{1}{\beta}\left(N-\frac{1}{2}\right) \ln N-\frac{N-1}{2 \beta} \ln \left(\frac{2 \pi}{\beta k}\right)$ does not depend on $u_{N}$ (see the details in [38]). Therefore, we introduce the free energy per particle

$$
F_{N}(x):=\frac{1}{N} E_{\mathrm{CG}}(x)
$$

so that

$$
\langle A\rangle_{N}=Z_{r}^{-1} \int_{\mathbb{R}} A\left(u^{N}\right) \exp \left(-\beta N F_{N}\left(u^{N}\right)\right) d u^{N} .
$$

The limit behaviour of $F_{N}$ is provided by the Large Deviations Principle. This claim is made precise in the following theorem. 
Theorem 2 Assume the potential $W$ satisfies

$$
\forall \xi \in \mathbb{R}, \quad \int_{\mathbb{R}} \exp (\xi y-\beta W(y)) d y<+\infty,
$$

and $\exp (-\beta W) \in H^{1}(\mathbb{R} \backslash\{0\})$. Then the limit behaviour of $F_{N}$ is given by the following Legendre transform:

$$
\lim _{N \rightarrow+\infty}\left(F_{N}(x)+\frac{1}{\beta} \ln \frac{z}{N}\right)=F_{\infty}(x)
$$

with

$$
F_{\infty}(x):=\frac{1}{\beta} \sup _{\xi}\left(\xi x-\ln \left[z^{-1} \int_{\mathbb{R}} \exp (\xi y-\beta W(y)) d y\right]\right)
$$

and $z=\int_{\mathbb{R}} \exp (-\beta W(y)) d y$.

Remark 4 The assumption $\exp (-\beta W) \in H^{1}(\mathbb{R} \backslash\{0\})$ allows for $W$ to be piecewise continuous, with discontinuity at the origin. This in particular allows to deal with the type of potentials mentioned in Remark 2.

Proof: Let us first rewrite the free energy $F_{N}(x)$ as follows:

$$
\begin{aligned}
F_{N}(x)= & -\frac{1}{\beta N} \ln \left[\int _ { \mathbb { R } ^ { N - 1 } } \operatorname { e x p } \left(-\beta \sum_{i=1}^{N-1} W\left(\frac{u^{i}-u^{i-1}}{h}\right)\right.\right. \\
& \left.\left.-\beta W\left(\frac{x-u^{N-1}}{h}\right)\right) d u^{1} \ldots d u^{N-1}\right] \\
= & -\frac{N-1}{\beta N} \ln h-\frac{1}{\beta N} \ln \left[\int _ { \mathbb { R } ^ { N - 1 } } \operatorname { e x p } \left(-\beta W\left(N x-\sum_{i=1}^{N-1} y_{i}\right)\right.\right. \\
& \left.\left.-\beta \sum_{i=1}^{N-1} W\left(y_{i}\right)\right) d y_{1} \ldots d y_{N-1}\right] \\
= & -\frac{1}{\beta} \ln h-\frac{1}{\beta} \ln z-\frac{1}{\beta N} \ln \mu_{N}(x),
\end{aligned}
$$

where $\mu_{N}$ is the law of the random variable $(1 / N) \sum_{i=1}^{N} Y_{i}$, and $Y_{i}$ is a sequence of i.i.d. random variables with law $\mu=z^{-1} \exp (-\beta W(y))$. Actually, we have

$$
\mu_{N}(x)=N \mu^{* N}(N x),
$$

where $\mu^{* N}$ denotes the $(N-1)$-fold convolution product of $\mu\left(\mu^{* 2}=\mu * \mu\right)$.

The sequence of measures $\mu_{N}$ satisfies a large deviations property (see for instance [23, $24,25,46])$. We are going to use it in order to compute the limit of $\frac{1}{N} \ln \mu_{N}$. We first prove a 
lower bound, which is a simple consequence of the results of [46]. The upper bound is more involved: we need to reproduce the corresponding proof of [46], and use a refined version of the Central Limit Theorem [32].

We introduce the function

$$
G_{N}(x)=-\frac{1}{\beta N} \ln \mu_{N}(x)
$$

which satisfies, in view of the above computation,

$$
F_{N}(x)=-\frac{1}{\beta} \ln \frac{z}{N}+G_{N}(x) .
$$

First step: lower bound. We write

$$
\mu_{N+1}(x)=(N+1) \int_{\mathbb{R}} \mu(N(x-t)+x) \mu_{N}(t) d t .
$$

Let us define

$$
J_{N}(t)=-\frac{1}{N} \ln \mu(N(x-t)+x) .
$$

This function clearly satisfies the following convergence:

$$
\liminf _{u \rightarrow t, N \rightarrow+\infty} J_{N}(u)=J_{\infty}(t):= \begin{cases}+\infty & \text { if } t \neq x \\ 0 & \text { if } t=x .\end{cases}
$$

Hence, we may apply Theorem 2.3 of [46], which implies that

$$
\liminf _{N \rightarrow+\infty}\left(-\frac{1}{N} \ln \int_{\mathbb{R}} \exp \left(-N J_{N}(t)\right) \mu_{N}(t) d t\right) \geq \inf _{t \in \mathbb{R}}\left(J_{\infty}(t)+\beta F_{\infty}(t)\right)=\beta F_{\infty}(x) .
$$

Since the left-hand side of (37) is equal to $\frac{\beta(N+1)}{N} G_{N+1}(x)+\frac{\ln (N+1)}{N}$, we infer

$$
\liminf _{N \rightarrow+\infty} G_{N}(x) \geq F_{\infty}(x)
$$

Second step: upper bound. We now aim at bounding $G_{N}$ from above. We recall that the function we maximize in (33) is concave, so there exists a unique $\xi_{x} \in \mathbb{R}$ such that

$$
F_{\infty}(x)=\frac{1}{\beta}\left(\xi_{x} x-\ln \left[z^{-1} \int_{\mathbb{R}} \exp \left(\xi_{x} y-\beta W(y)\right) d y\right]\right) .
$$

The Euler-Lagrange equation of the maximization problem also implies

$$
x=\frac{\int_{\mathbb{R}} y \exp \left(\xi_{x} y-\beta W(y)\right) d y}{\int_{\mathbb{R}} \exp \left(\xi_{x} y-\beta W(y)\right) d y} .
$$


We introduce the notations

$$
\tilde{\mu}(t)=\frac{\exp \left(\xi_{x} t-\beta W(t)\right)}{\int_{\mathbb{R}} \exp \left(\xi_{x} t-\beta W(t)\right) d t} \text { and } M(\xi)=z^{-1} \int_{\mathbb{R}} \exp (\xi t-\beta W(t)) d t,
$$

and compute

$$
\begin{aligned}
\mu_{N}(x)= & N \int_{\mathbb{R}^{N-1}} \mu\left(N x-\sum_{i=1}^{N-1} y_{i}\right) \mu\left(y_{1}\right) \ldots \mu\left(y_{N-1}\right) d y_{1} \ldots d y_{N-1} \\
= & N M\left(\xi_{x}\right)^{N-1} \int_{\mathbb{R}^{N-1}} \mu\left(N x-\sum_{i=1}^{N-1} y_{i}\right) \exp \left(-\xi_{x} \sum_{i=1}^{N-1} y_{i}\right) \\
& \left.\times \tilde{\mu}\left(y_{1}\right) \ldots \tilde{\mu}\left(y_{N-1}\right) d y_{1} \ldots d y_{N-1}\right) \\
\geq & \left.N M\left(\xi_{x}\right)^{N-1} \int_{\left|N x-\sum y_{i}\right| \leq \delta}^{N-1}\right) \\
& \times \exp \left(-\xi_{x} \sum_{i=1}^{N-1} y_{i}\right) \tilde{\mu}\left(y_{1}\right) \ldots \tilde{\mu}\left(y_{N-1}\right) d y_{1} \ldots d y_{N-1} \\
\geq & N M\left(\xi_{x}\right)^{N-1}\left(\inf _{[-\delta, \delta]} \mu\right) \exp \left(-\xi_{x} N x-\left|\xi_{x}\right| \delta\right) \\
& \times \int_{\left|N x-\sum y_{i}\right| \leq \delta} \tilde{\mu}\left(y_{1}\right) \ldots \tilde{\mu}\left(y_{N-1}\right) d y_{1} \ldots d y_{N-1} .
\end{aligned}
$$

Hence,

$$
\begin{aligned}
G_{N}(x) \leq & -\frac{1}{\beta N} \ln N-\frac{N-1}{\beta N} \ln \left(M\left(\xi_{x}\right)\right)+\frac{\xi_{x} x}{\beta}+\left|\xi_{x}\right| \frac{\delta}{\beta N}-\frac{1}{\beta N} \ln \left(\inf _{[-\delta, \delta]} \mu\right) \\
& -\frac{1}{\beta N} \ln \mathbb{P}\left(\left|\frac{1}{N} \sum_{i=1}^{N-1} Y_{i}-x\right| \leq \frac{\delta}{N}\right)
\end{aligned}
$$

where the random variables $Y_{i}$ are i.i.d. of law $\tilde{\mu}$. The equation (39) implies that $\mathbb{E}\left(Y_{i}\right)=$ $x$. According to the hypotheses on $W$, we have $\tilde{\mu} \in H^{1}(\mathbb{R} \backslash\{0\})$, hence we may apply Theorem 5.1 of [32]. It implies that the law $\theta_{N}$ of the variable $\left(\sum_{i=1}^{N} Y_{i}-N x\right) / \sqrt{N}$ converges in $H^{1}(\mathbb{R})$ to some normal law. In particular, we have convergence in $L^{\infty}$, hence

$$
\mathbb{P}\left(\left|\frac{1}{N} \sum_{i=1}^{N-1} Y_{i}-x\right| \leq \frac{\delta}{N}\right)=\int_{\frac{x-\delta}{\sqrt{N-1}}}^{\frac{x+\delta}{\sqrt{N-1}}} \theta_{N-1}(t) d t \geq \frac{2 \gamma \delta}{\sqrt{N-1}}
$$


for $N$ large enough, where $\gamma>0$ does not depend on $N$. Inserting this inequality into (40), we find

$$
\begin{aligned}
G_{N}(x) \leq & -\frac{1}{\beta N} \ln N-\frac{N-1}{\beta N} \ln \left(M\left(\xi_{x}\right)\right)+\frac{\xi_{x} x}{\beta}+\left|\xi_{x}\right| \frac{\delta}{\beta N}-\frac{1}{\beta N} \ln \left(\inf _{[-\delta, \delta]} \mu\right) \\
& -\frac{1}{\beta N} \ln \left(\frac{2 \gamma \delta}{\sqrt{N-1}}\right) .
\end{aligned}
$$

Hence,

$$
\limsup _{N \rightarrow+\infty} G_{N}(x) \leq-\frac{1}{\beta} \ln \left(M\left(\xi_{x}\right)\right)+\frac{\xi_{x} x}{\beta},
$$

which implies, according to the definition of $M$ and $\xi_{x}$, that

$$
\limsup _{N \rightarrow+\infty} G_{N}(x) \leq F_{\infty}(x) .
$$

Estimates (38) and (42) imply $\lim _{N \rightarrow+\infty} G_{N}(x)=F_{\infty}(x)$. In view of (35), this implies (32). $\diamond$

Remark 5 (The small temperature limit) As in Remark 3, it is possible to compute the expansion of $F_{\infty}(x)$ as $\beta \rightarrow+\infty$. Using the Laplace method, and assuming that $W$ is convex, one finds that

$$
F_{\infty}(x)=W(x)+\frac{1}{2 \beta} \ln W^{\prime \prime}(x)+O\left(\frac{1}{\beta^{2}}\right) .
$$

Let us now consider another strategy to find an approximation of $F_{N}$. In the spirit of the QuasiContinuum Method, we expand $E_{\mu}\left(u^{1}, \ldots, u^{N}\right)$ around the equilibrium configuration $\bar{u}^{i}=i u^{N} / N$, for a given $u^{N}$. More precisely, we set $u^{i}=\bar{u}^{i}+\xi_{i}$, assume that $\xi_{i}$ is small, and expand the energy at second order with respect to $\xi_{i}$, as explained in the Introduction (see (11)). We next insert this approximated energy $\widetilde{E}$ in (29). Due to the harmonic approximation, the resulting coarse-grained energy, that we denote $E_{\mathrm{QCM}}$, is analytically computable and writes

$$
E_{\mathrm{QCM}}(x)=N W(x)+\frac{N-1}{2 \beta} \ln W^{\prime \prime}(x)+\frac{N-1}{2 \beta} \ln \frac{\beta}{2 \pi}+\frac{1}{2 \beta} \ln N .
$$

Hence,

$$
F_{\mathrm{QCM}}(x):=\lim _{N \rightarrow+\infty} \frac{1}{N} E_{\mathrm{QCM}}(x)=W(x)+\frac{1}{2 \beta} \ln W^{\prime \prime}(x)+\frac{1}{2 \beta} \ln \frac{\beta}{2 \pi} .
$$

Thus, up to a constant, $F_{\mathrm{QCM}}(x)$ corresponds to the first-order approximation (in powers of $1 / \beta)$ of $F_{\infty}(x)$.

Slightly improving the proof of Theorem 2 above, it is also possible to prove the convergence of the derivative of the free energy, a quantity which is indeed practically relevant (e.g. for the simulation of (10)): 
Corollary 1 Assume that the hypotheses of Theorem 2 are satisfied. Then, we have

$$
F_{N}(x)+\frac{1}{\beta} \ln \left(\frac{z}{N}\right) \longrightarrow F_{\infty}(x) \quad \text { in } \quad L_{\mathrm{loc}}^{p}, \quad \forall p \in[1,+\infty) .
$$

In particular, this implies that $F_{N}^{\prime}$ converges to $F_{\infty}^{\prime}$ in $W_{\mathrm{loc}}^{-1, p}$.

Proof: According to Theorem 2, we already know the pointwise convergence of $G_{N}(x)=$ $F_{N}(x)+\beta^{-1} \ln (z / N)$. We therefore only need to prove that $G_{N}$ is bounded in $L_{\text {loc }}^{\infty}$ to prove our claim.

Lower bound: We go back to (36), and point out that $\mu \leq 1 / z$. Hence,

$$
\mu_{N+1}(x) \leq \frac{N+1}{z} \int_{\mathbb{R}} \mu_{N}=\frac{N+1}{z},
$$

which implies, using (34), that

$$
G_{N+1}(x) \geq-\frac{1}{\beta(N+1)} \ln \frac{N+1}{z}
$$

which is bounded from below independently of $N$.

Upper bound: We return to (41), and notice that according to the definition of $\xi_{x}$, the function $x \mapsto \xi_{x}$ is continuous. In addition, the constant $\gamma$ in (41) is a continuous function of $\xi_{x}$. Therefore, (41) provides an upper bound on $G_{N}$.

As a conclusion, $G_{N}$ is bounded in $L_{\text {loc }}^{\infty}$, which allows to conclude.

Remark 6 Considering the above theoretical results, it could be tempting to approach the average (30), that is,

$$
\langle A\rangle_{N}=Z_{r}^{-1} \int_{\mathbb{R}} A\left(u^{N}\right) \exp \left(-\beta N F_{N}\left(u^{N}\right)\right) d u^{N}
$$

by

$$
Z_{\infty}^{-1} \int_{\mathbb{R}} A\left(u^{N}\right) \exp \left(-\beta N F_{\infty}\left(u^{N}\right)\right) d u^{N}
$$

Note that $F_{N}$ has been replaced by $F_{\infty}$ in the exponential factor. This strategy is not efficient since this approximation does not provide the expansion (22)-(23) of $\langle A\rangle_{N}$ in powers of $1 / N$. Indeed, it is possible to use the Laplace method to compute the expansion of (46) as $N \rightarrow+\infty$. It reads

$$
A\left(y^{*}\right)+\frac{1}{2 N}\left(\sigma^{2} A^{\prime \prime}\left(y^{*}\right)+\frac{d_{3}}{\sigma^{2}} A^{\prime}\left(y^{*}\right)\right)+o\left(\frac{1}{N}\right),
$$

where $\sigma$ is defined by (23) and $d_{3}=z^{-1} \int_{\mathbb{R}}\left(y-y^{*}\right)^{3} \exp (-\beta W(y)) d y$. This expansion coincides with (22)-(23) only for the first term, that is $A\left(y^{*}\right)$. The second one differs, unless $d_{3} A^{\prime}\left(y^{*}\right)=0$. 
To improve the approximation (46), one may use the precised large deviations principle (see [16, Th. 3.7.4] or [4]). In such a case, one replaces (46) by

$$
Z_{\infty}^{-1} \int_{\mathbb{R}} A\left(u^{N}\right) \sqrt{F_{\infty}^{\prime \prime}\left(u^{N}\right)} \exp \left(-\beta N F_{\infty}\left(u^{N}\right)\right) d u^{N}
$$

This quantity is well-defined since $F_{\infty}$ is a convex function. Then it is seen that the expansion of (47) in powers of $1 / N$ agrees with (22)-(23) up to the second term. Note however that using (47) leads to a much more expensive computation than using (23).

The above convergence of the free energy $F_{N}$ is useful e.g. for the computation of the free energy of a chain of atoms with a prescribed length. Indeed, in such a case, we impose

$$
u^{N}=\ell
$$

where $\ell$ is fixed, and aim at computing the free energy $F_{N}$ as a function of $\ell$, in the limit $N \rightarrow+\infty$. We have

$$
F_{N}(\ell)=-\frac{1}{\beta N} \ln \left[\int_{\mathbb{R}^{N-1}} \exp \left(-\beta \sum_{i=1}^{N} W\left(\frac{u^{i}-u^{i-1}}{h}\right)\right) d u^{1} \ldots d u^{N-1}\right],
$$

where $u^{N}=\ell$. The limit of $F_{N}$ is provided by Theorem 2 .

Another interest of the approach is to provide an approximation of $F_{N}^{\prime}(\ell)$, a quantity related to the constitutive law of the material under consideration, at the finite temperature $1 / \beta$. Indeed, note that

$$
F_{N}^{\prime}(\ell)=\frac{\left\langle A_{N}\right\rangle_{N-1}}{\left\langle B_{N}\right\rangle_{N-1}}
$$

where $\langle\cdot\rangle_{N-1}$ is the average with respect to the Gibbs measure associated to the energy $\sum_{i=1}^{N-1} W\left(\frac{u^{i}-u^{i-1}}{h}\right)$, and the observables $A_{N}$ and $B_{N}$ are defined by

$$
\begin{aligned}
& B_{N}\left(u^{1}, \ldots, u^{N-1}\right)=\exp \left(-\beta W\left(N\left(\ell-u^{N-1}\right)\right)\right) \\
& A_{N}\left(u^{1}, \ldots, u^{N-1}\right)=W^{\prime}\left(N\left(\ell-u^{N-1}\right)\right) \exp \left(-\beta W\left(N\left(\ell-u^{N-1}\right)\right)\right) .
\end{aligned}
$$

Hence $F_{N}^{\prime}(\ell)$ can be interpreted as the average force between atoms $N-1$ and $N$, when the positon of atom $N$ is prescribed at $u^{N}=\ell$. Corollary 1 provides the convergence of $F_{N}^{\prime}(\ell)$ to $F_{\infty}^{\prime}(\ell)$ in a weak norm.

Remark 7 Note that, in (48), both observables $A_{N}$ and $B_{N}$ depend on $N$. Hence, the results of Section 2.1 (obtained using the Law of Large Numbers and not involving the Large Deviations Principle) do not apply to compute the large $N$ limit of $\left\langle A_{N}\right\rangle_{N-1}$ and $\left\langle B_{N}\right\rangle_{N-1}$. 


\subsection{Numerical tests}

For our numerical tests, we choose the pair interaction potential

$$
W(x)=\frac{1}{2}(x-1)^{4}+\frac{1}{2} x^{2}
$$

shown on Figure 4. Note that $W(x)$ grows fast enough to $+\infty$ when $|x| \rightarrow+\infty$, such that assumptions (20) and (31) are satisfied. Note also that we have made no assumption on the convexity of $W$ in Theorems 1 and 2. We consider here a convex potential. At the end of this section, we will consider a non-convex example (see (50)), and show that we obtain similar conclusions.

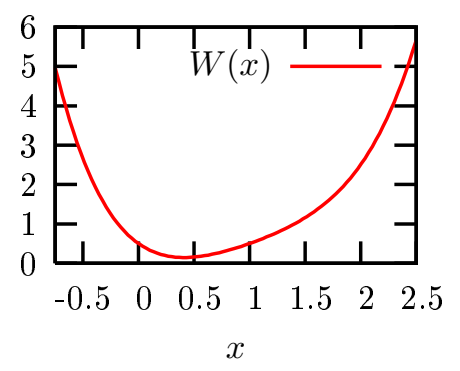

Figure 4: The potential $W$ chosen for the tests.

We first consider the computation of ensemble averages, and we again restrict ourselves to the case of two repatoms $u^{0}=0$ and $u^{N}$. This is just for simplicity and for the sake of demonstrating the feasibility and the interest of our approach. The case of $N_{r}$ repatoms may be treated likewise. It is of course more computationally demanding, although affordable.

We choose an observable $A(x)$, and we compare the following four quantities:

(i) the exact average $\langle A\rangle_{N}$ defined by (14). Following (4)-(5), this quantity is computed as the long-time average of $A\left(u^{N}(t)\right)$ along the full system dynamics

$$
d u=-\nabla_{u} E_{\mu}(u) d t+\sqrt{2 / \beta} d B_{t} \quad \text { in } \mathbb{R}^{N}
$$

This equation is numerically integrated with the forward Euler scheme, with a small time step. In practice, we have simulated many independent realizations of this SDE, in order to compute error bars for $\langle A\rangle_{N}$.

(ii) a QuasiContinuum type approximation of $\langle A\rangle_{N}$, based on the 'interpolation + harmonic expansion' procedure outlined above. That is, we introduce $E_{\mathrm{QCM}}$ defined by 
(43), and we approximate $\langle A\rangle_{N}$ by

$$
\langle A\rangle_{N}^{\mathrm{QCM}}:=\frac{\int_{\mathbb{R}} A(x) \exp \left[-\beta E_{\mathrm{QCM}}(x)\right] d x}{\int_{\mathbb{R}} \exp \left[-\beta E_{\mathrm{QCM}}(x)\right] d x} .
$$

(iii) a Law of Large Numbers (LLN) type approximation of $\langle A\rangle_{N}$, which consists in approximating $\langle A\rangle_{N}$ by $A\left(y^{*}\right)$, following Theorem 1 .

(iv) a refined approximation, which consists in approximating $\langle A\rangle_{N}$ by $\langle A\rangle_{N}^{\text {approx,1 }}$ defined by (23), following Theorem 1 .

Note that only one-dimensional integrals are needed for approximations (ii), (iii) and (iv). They can be computed with a high accuracy.

We plot on Figure 5 these four quantities, for increasing values of $N$ (the temperature is fixed at $1 / \beta=1$ ), for the observable $A(x)=\exp (x)$. On Figure 6 , we compare the same quantities, now as functions of the temperature, for $N=100$ and for $N=10$. We here work with $A(x)=x^{2}$, for which $\langle A\rangle_{N}=\langle A\rangle_{N}^{\text {approx }, 1}$.
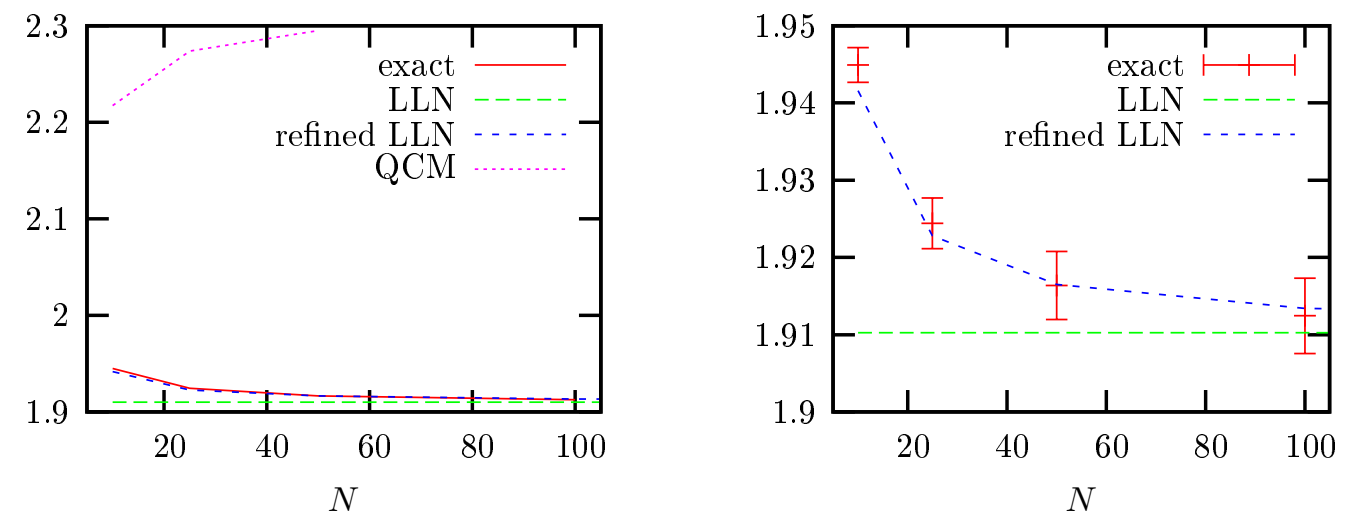

Figure 5: Convergence, as $N$ increases, of $\left\langle A\left(u^{N}\right)\right\rangle_{N}$ (exact), of $\left\langle A\left(u^{N}\right)\right\rangle_{N}^{\text {approx, } 1}$ (refined LLN) and of $\left\langle A\left(u^{N}\right)\right\rangle_{N}^{\mathrm{QCM}}(\mathrm{QCM})$ and comparison to $A\left(y^{*}\right)(\mathrm{LLN})$ (temperature $1 / \beta=1$, observable $A(x)=\exp (x)$; we have performed computations for $N=10,25,50$ and 100; on the right graph, we show error bars for $\left.\left\langle A\left(u^{N}\right)\right\rangle_{N}\right)$.

As expected, the thermodynamic limit strategies (iii) and (iv) better agree with the full atom calculation, whatever the temperature, provided the number of eliminated atoms is large (note that the strategy (iv) is very accurate even for the small value $N=10$, at the temperature $1 / \beta=1$ ). Approximation (ii) is clearly ineffective for high temperatures. On the other hand, for a sufficiently small temperature and a sufficiently small number of 

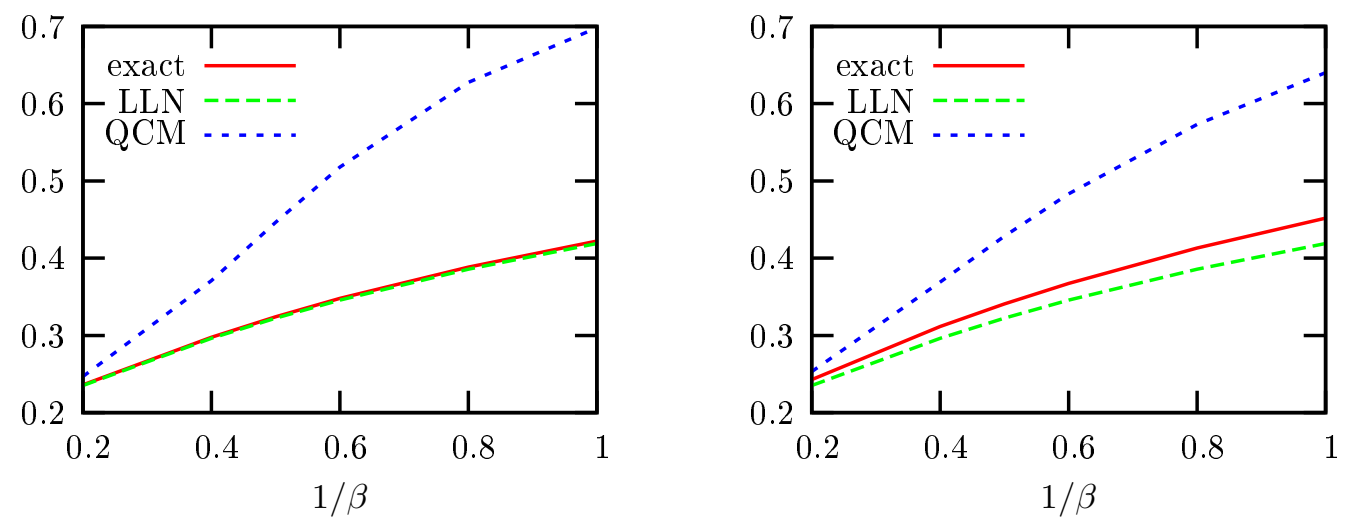

Figure 6: We plot $\left\langle A\left(u^{N}\right)\right\rangle_{N}=\left\langle A\left(u^{N}\right)\right\rangle_{N}^{\text {approx,1 }}$ (exact), $\left\langle A\left(u^{N}\right)\right\rangle_{N}^{\mathrm{QCM}}(\mathrm{QCM})$ and $A\left(y^{*}\right)$ (LLN) as functions of the temperature $1 / \beta$ : on the left, $N=100$; on the right, $N=10$ (observable $A(x)=x^{2}$ ).

eliminated atoms, this approximation is close to the full atom result. However, even for the small values $N=10$ and $1 / \beta=0.2$, our asymptotic result $\left\langle A\left(u^{N}\right)\right\rangle_{N}^{\text {approx, } 1}=1.6299$ (for $A(x)=\exp (x))$ is closer to the exact result $\left\langle A\left(u^{N}\right)\right\rangle_{N}=1.6303 \pm 0.0008$ than the QCM result $\left\langle A\left(u^{N}\right)\right\rangle_{N}^{\mathrm{QCM}}=1.6469$.

Remark 8 As in Remark 2, we emphasize that the computations reported on here do not account for the constraints on the positions of atoms. Analogous computations, that account for constraints, may be performed. They provide similar conclusions, as can be seen on Figure 7, which is very similar to Figure 6.

We now consider the computation of free energies, more precisely, of the derivatives of free energies. The full atom value $F_{N}^{\prime}(x)$ is computed as a ratio of ensemble averages (see (48)). We compare this quantity with

(i) its large $N$ limit $F_{\infty}^{\prime}(x)$, where $F_{\infty}$ is defined by (33), on the one hand,

(ii) and, on the other hand, its QuasiContinuum type approximation $F_{\mathrm{QCM}}^{\prime}(x)$, where $F_{Q C M}$ is defined by (44). It reads

$$
F_{\mathrm{QCM}}^{\prime}(x)=W^{\prime}(x)+\frac{1}{2 \beta} \frac{W^{\prime \prime \prime}(x)}{W^{\prime \prime}(x)} .
$$

We briefly detail how we compute $F_{\infty}^{\prime}(x)$. Let $\xi_{x}$ be the unique real number at which the supremum in (33) is attained. We have

$$
F_{\infty}^{\prime}(x)=\frac{\xi_{x}}{\beta} .
$$




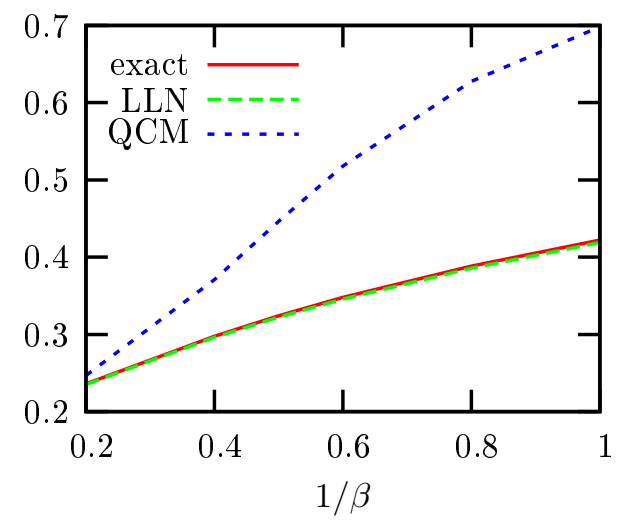

Figure 7: We plot $\left\langle A\left(u^{N}\right)\right\rangle_{N}=\left\langle A\left(u^{N}\right)\right\rangle_{N}^{\text {approx,1 }}$ (exact), $\left\langle A\left(u^{N}\right)\right\rangle_{N}^{\mathrm{QCM}}$ (QCM) and $A\left(y^{*}\right)$ (LLN) as functions of the temperature $1 / \beta\left(N=100\right.$, observable $\left.A(x)=x^{2}\right)$. The potential energy is of type (18): it is equal to $W(x)$ defined by (49) if $x>0$, and $+\infty$ otherwise.

The Euler-Lagrange equation solved by $\xi_{x}$ is (39), that we recast as

$$
z^{-1} \int_{\mathbb{R}}(x-y) \exp \left(\xi_{x} y\right) \exp (-\beta W(y)) d y=0 .
$$

Let us introduce $G(y, \xi)=(x-y) \exp (\xi y)$. We hence look for $\xi_{x}$ such that $\mathbb{E}_{\mu}\left[G\left(y, \xi_{x}\right)\right]=0$, where the random variable $y$ is distributed according to the probability measure $\mu(y)=$ $z^{-1} \exp (-\beta W(y))$. The Robbins-Monroe algorithm [28] can be used to compute $\xi_{x}$, hence $F_{\infty}^{\prime}(x)$.

We first study the convergence of $F_{N}^{\prime}(x)$ to $F_{\infty}^{\prime}(x)$ as $N$ increases, for a fixed chain length $x=1.4$ and a fixed temperature $1 / \beta=1$. Results are shown on Figure 8 . We indeed observe that $F_{N}^{\prime}(x) \rightarrow F_{\infty}^{\prime}(x)$ when $N \rightarrow+\infty$.

We now compare the two approximations (i) and (ii) of $F_{N}^{\prime}(x)$, for $N=100$ and $1 / \beta=1$. Results are shown on Figure 9. We observe that $F_{\infty}^{\prime}(x)$ is a very good approximation of $F_{N}^{\prime}(x)$. As expected, the temperature is too high for the harmonic approximation to provide an accurate approximation of $F_{N}^{\prime}(x)$.

On Figure 10, we plot $F_{\infty}^{\prime}(x)$ for several temperatures, as well as its zero temperature limit, which is $W^{\prime}(x)$ (see Remark 5).

Up to here, we have used the convex potential (49). For the sake of completeness, we now briefly consider the case of a non-convex potential $W$. We choose the toy-model

$$
W(x)=\left(x^{2}-1\right)^{2},
$$

which corresponds to a double-well potential. On Figure 11, we plot $F_{\infty}^{\prime}(x)$ for several temperatures, for this double-well potential. Although we have not yet compared these 


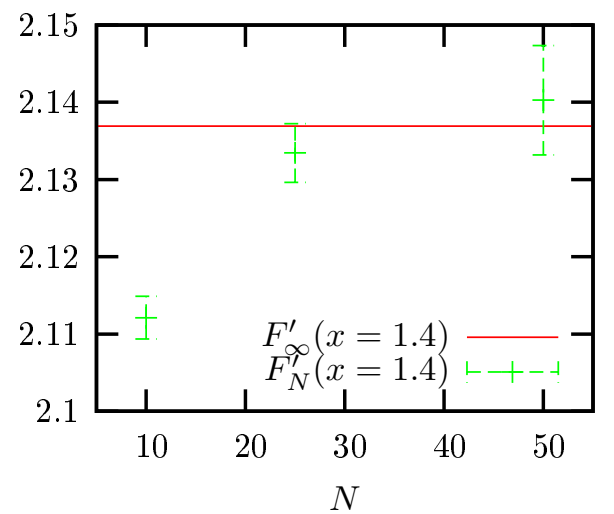

Figure 8: Convergence of $F_{N}^{\prime}(x)$ (shown with error bars) to $F_{\infty}^{\prime}(x)$ as $N$ increases (temperature $1 / \beta=1$, fixed chain length $x=1.4$ ).

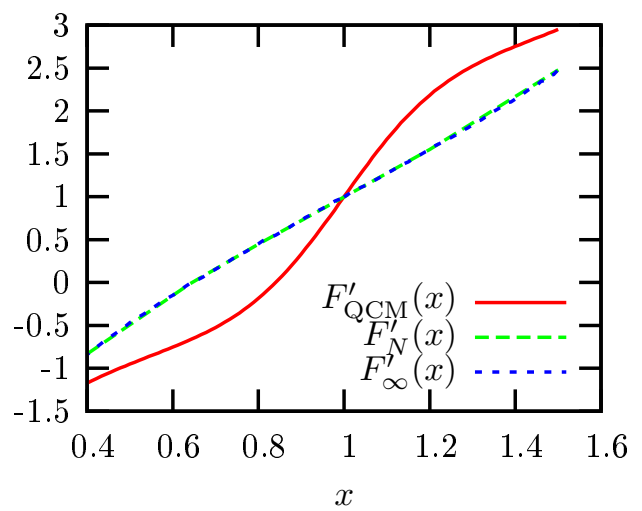

Figure 9: We plot $F_{N}^{\prime}(x), F_{\infty}^{\prime}(x)$ and $F_{\mathrm{QCM}}^{\prime}(x)$, for the temperature $1 / \beta=1$ and $N=100$. 


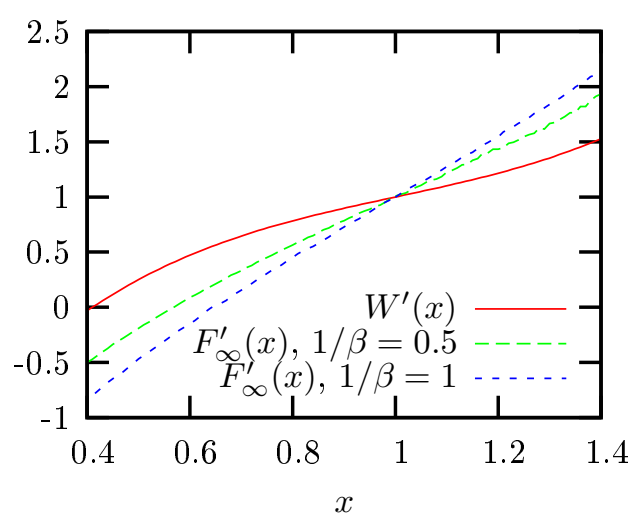

Figure 10: $F_{\infty}^{\prime}(x)$ for different temperatures.

coarse-grained computations with the full atom computations, the numerical results reported here are consistent with the small temperature limit $\lim _{T \rightarrow 0} F_{\infty}^{\prime}(x)=\left(W^{*}\right)^{\prime}(x)$, where $W^{*}$ is the convex envelop of $W$.

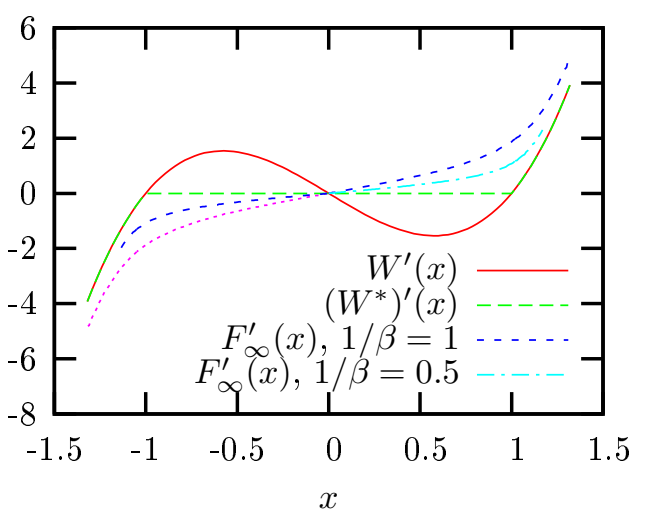

Figure 11: $F_{\infty}^{\prime}(x)$ for different temperatures, in the case of the double-well potential (50).

\section{The NNN case and some extensions}

In this Section, we first consider the case of a NNN interacting system. The analysis is detailed in Section 3.1. In Section 3.2, we point out some possible extensions, first the 
NNNN case (still for one-dimensional systems) and second the case of linear polymer chains, where atoms sample the physical space $\mathbb{R}^{3}$.

\subsection{The next-to-nearest neighbour (NNN) case}

We now consider the next-to-nearest neighbour case. It turns out that, for the computation of ensemble averages as well as for other questions, this case is significantly more intricate than the NN case. Our strategy, based on the Law of Large Numbers, will be similar to that used for the NN case, but the object manipulated are not independent random variables any longer. Markov chains are the right notion formalizing the situation mathematically.

We begin by introducing the rescaled atomistic energy, similarly to (13):

$$
E_{\mu}\left(u^{1}, \ldots, u^{N}\right)=\sum_{i=1}^{N} W_{1}\left(\frac{u^{i}-u^{i-1}}{h}\right)+\sum_{i=1}^{N-1} W_{2}\left(\frac{u^{i+1}-u^{i-1}}{h}\right) .
$$

As above, we introduce the change of variables (15), replacing $\left(u^{i}-u^{i-1}\right) / h$ by the interatomic distances $y_{i}$. Recall from (16) that $u^{N}=\frac{1}{N} \sum_{i=1}^{N} y_{i}$. The ensemble average $\langle A\rangle_{N}$ of an observable that depends only on the right-end atom therefore writes

$$
\begin{aligned}
\langle A\rangle_{N} & =Z^{-1} \int_{\mathbb{R}^{N}} A\left(u^{N}\right) \exp \left(-\beta E_{\mu}\left(u^{1}, \ldots, u^{N}\right)\right) d u^{1} \ldots d u^{N} \\
& =Z^{-1} \int_{\mathbb{R}^{N}} A\left(\frac{1}{N} \sum_{i=1}^{N} y_{i}\right) e^{-\beta \sum_{i} W_{1}\left(y_{i}\right)} e^{-\beta \sum_{i} W_{2}\left(y_{i}+y_{i+1}\right)} d y_{1} \ldots d y_{N} .
\end{aligned}
$$

The key ingredient is now to see the above expression, as $N$ goes to infinity, as an asymptotics for a discrete-time Markov chain. The asymptotics of Markov chains being a mathematical problem much more involved than that of i.i.d. sequences, we restrict ourselves to the computation of the average of an observable. The asymptotic behaviour of the free energy may be studied, applying a Large Deviations Principle for Markov chains (see for instance [26, Th. IV.3]). We will not pursue in this direction.

Section 3.1.1 deals with the case of two repatoms (namely $u^{0}=0$ and $u^{N}$ ), while Section 3.1.2 indicate the changes in order to deal with more than two repatoms. Numerical results will be reported in Section 3.1.3.

\subsubsection{Limit of the average, the case of two repatoms}

In order to compute $\lim _{N \rightarrow+\infty}\langle A\rangle_{N}$, we introduce the notation

$$
f(x, y):=\exp \left(-\beta W_{2}(x+y)\right) \exp \left(-\beta W_{1}(y)\right) .
$$


Equation (52) rewrites

$$
\langle A\rangle_{N}=Z^{-1} \int_{\mathbb{R}^{N}} A\left(\frac{1}{N} \sum_{i=1}^{N} y_{i}\right) e^{-\beta W_{1}\left(y_{1}\right)} f\left(y_{1}, y_{2}\right) \ldots f\left(y_{N-1}, y_{N}\right) d y_{1} \ldots d y_{N} .
$$

Our method consists in considering the sequence of variables $\left(y_{1}, \ldots, y_{N}\right)$ in $(53)$ as a realization of a Markov chain with kernel $f(\cdot, \cdot)$. However, the slight technical difficulty at this stage is that the kernel $f$ is not normalized, since in general

$$
\int_{\mathbb{R}} f\left(y_{1}, y_{2}\right) d y_{2}=\int_{\mathbb{R}} \exp \left(-\beta W_{2}\left(y_{1}+y_{2}\right)\right) \exp \left(-\beta W_{1}\left(y_{2}\right)\right) d y_{2} \neq 1 .
$$

A standard trick of Probability theory allows to circumvent this difficulty. Introduce

$$
\bar{f}(x, y):=\exp \left[-\beta W_{2}(x+y)-\frac{\beta}{2} W_{1}(x)-\frac{\beta}{2} W_{1}(y)\right] .
$$

Note that $\bar{f}$ is a symmetric function (whereas $f$ is not), hence the operator

$$
P \phi(y)=\int_{\mathbb{R}} \bar{f}(y, z) \phi(z) d z
$$

is self-adjoint on $L^{2}(\mathbb{R})$. Consider then

$$
\psi_{1}:=\operatorname{argmax}\left\{\int_{\mathbb{R}^{2}} \psi(y) \psi(z) \bar{f}(y, z) d y d z ; \int_{\mathbb{R}} \psi^{2}(y) d y=1\right\},
$$

and set

$$
\lambda=\int_{\mathbb{R}^{2}} \psi_{1}(y) \psi_{1}(z) \bar{f}(y, z) d y d z .
$$

Using standard tools of spectral theory of self-adjoint compact operators, it is possible to prove that the eigenvalue $\lambda$ and the eigenvector $\psi_{1}$ exist, and that, up to changing $\psi_{1}$ into $-\psi_{1}$, they are unique. In addition, one can choose $\psi_{1}$ such that $\psi_{1}>0$. They satisfy

$$
\lambda \psi_{1}(y)=\int_{\mathbb{R}} \bar{f}(y, z) \psi_{1}(z) d z
$$

We now define

$$
g(y, z):=\frac{\psi_{1}(z)}{\lambda \psi_{1}(y)} \bar{f}(y, z)
$$

By construction,

$$
\int_{\mathbb{R}} g(y, z) d z=1, \quad \int_{\mathbb{R}} \psi_{1}^{2}(y) g(y, z) d y=\psi_{1}^{2}(z) .
$$


The average (53) now reads

$$
\begin{aligned}
\langle A\rangle_{N}= & Z^{-1} \int_{\mathbb{R}^{N}} A\left(\frac{1}{N} \sum_{i=1}^{N} y_{i}\right) \psi_{1}\left(y_{1}\right) e^{-\frac{\beta}{2} W_{1}\left(y_{1}\right)} g\left(y_{1}, y_{2}\right) \ldots g\left(y_{N-1}, y_{N}\right) \\
& \frac{e^{-\frac{\beta}{2} W_{1}\left(y_{N}\right)}}{\psi_{1}\left(y_{N}\right)} d y
\end{aligned}
$$

where $\left(y_{1}, \ldots, y_{N}\right)$ may now be seen as a realization of a normalized Markov chain of kernel $g$, with invariant probability measure $\psi_{1}^{2}$. We assume that $\bar{f}$ decays fast enough at infinity (which ensures for instance that (55) is well-posed) and that it is positive. This latter assumption ensures that the Markov chain satisfies the following accessibility condition: for all $x \in \mathbb{R}$, and for all measurable set $\mathcal{B} \subset \mathbb{R}$ of positive Lebesgue measure, we have

$$
\int_{\mathcal{B}} g(x, y) d y>0 .
$$

Under this property, combined with the existence of an invariant probability measure, it is known (see [33, Th. 17.1.7]) that the Markov chain satisfies a Law of Large Numbers with respect to the measure $\psi_{1}^{2}$. We now state a direct corollary of this general result that applies to our context.

Theorem 3 Assume that $A$ is continuous, and satisfies the following conditions:

$$
\exists p \geq 0, \quad \exists C>0, \quad \forall x \in \mathbb{R}, \quad|A(x)| \leq C\left(1+|x|^{p}\right) .
$$

Under the assumptions that $W_{1}, W_{2} \in L_{\text {loc }}^{1}(\mathbb{R})$ are bounded from below, that $e^{-\beta W_{1}}, e^{-\beta W_{2}} \in$ $W_{\text {loc }}^{1,1}(\mathbb{R})$ and that

$$
\forall q \geq 0, \int_{\mathbb{R}}|x|^{q} e^{-\beta W_{1}(x)} d x<+\infty \quad \text { and } \quad \int_{\mathbb{R}}|x|^{q} e^{-\beta W_{2}(x)} d x<+\infty,
$$

the ergodic theorem for Markov chains [33] yields

$$
\lim _{N \rightarrow+\infty}\langle A\rangle_{N}=A\left(y^{*}\right)
$$

where

$$
y^{*}:=\int_{\mathbb{R}} y \psi_{1}^{2}(y) d y
$$

Remark 9 Note that, for the result to hold true, (58) is not needed. The existence of the moment of order $p$ is sufficient. However, assumption (58) will be useful for Theorem 4 below. 
Remark 10 It might sound a little strange that $\psi_{1}$ is the eigenvector of the transition operator $P$ defined by (54), whereas the invariant measure of the chain is $\psi_{1}^{2}$. This is explained by the following fact: the expectation value of $y_{i}$ is equal to

$$
\mathbb{E}\left(y_{i}\right)=\int_{\mathbb{R}} x\left(P^{i} \varphi_{0}\right)(x)\left(P^{N-i} \varphi_{1}\right)(x) d x
$$

for some initial laws $\varphi_{0}$ and $\varphi_{1}$. Hence, if $1 \ll i \ll N$, then $P^{i} \varphi_{0}$ and $P^{N-i} \varphi_{1}$ converge to the eigenvector of $P$ associated with the largest eigenvalue, that is, $\psi_{1}$. This is explained in more details in Section 3.2.1 below in the case of a non self-adjoint transition operator P.

Here again, it is possible to compute the next terms in the expansion of $\langle A\rangle_{N}$ in powers of $1 / N$. However, the computations are much more intricate than in the i.i.d. case. The terms of the expansion here contain covariance terms, together with terms containing the initial state of the Markov chain. We give as an example the first term of the expansion in the following theorem.

Theorem 4 Assume that $A$ is of class $C^{3}$. Then, under the assumptions of Theorem 3, we have

$$
\begin{aligned}
\langle A\rangle_{N}= & A\left(y^{*}\right)+\frac{1}{N} A^{\prime}\left(y^{*}\right) \sum_{i \geq 1} \mathbb{E}\left(Y_{i}-y^{*}\right) \\
& +\frac{1}{N} A^{\prime \prime}\left(y^{*}\right)\left[\frac{1}{2} \int_{\mathbb{R}}\left(x-y^{*}\right)^{2} \psi_{1}^{2}(x) d x\right. \\
& \left.+\sum_{i \geq 2} \mathbb{E}\left(\left(\tilde{Y}_{i}-y^{*}\right)\left(\tilde{Y}_{1}-y^{*}\right)\right)\right]+O\left(\frac{1}{N^{2}}\right),
\end{aligned}
$$

where $\left(Y_{i}\right)_{i \geq 1}$ and $\left(\tilde{Y}_{i}\right)_{i \geq 1}$ are Markov chains of initial law $Z_{1}^{-1} \psi_{1} e^{-\frac{\beta}{2} W_{1}}$ and $\psi_{1}^{2}$ respectively, and of transition kernel $g$. Moreover, the series appearing in (59) converge exponentially fast.

Remark 11 Let us mention that the term multiplying $A^{\prime \prime}\left(y^{*}\right)$ is exactly the variance appearing in the Central Limit Theorem for Markov chains [33, Th. 17.0.1], namely:

$$
\sigma^{2}=\int_{\mathbb{R}}\left(x-y^{*}\right)^{2} \psi_{1}^{2}(x) d x+2 \sum_{i \geq 2} \mathbb{E}\left(\left(\tilde{Y}_{i}-y^{*}\right)\left(\tilde{Y}_{1}-y^{*}\right)\right)
$$

In addition, we see that in the special case of i.i.d. random variables, the second term of the right-hand side of (59) vanishes, together with the last one. We then recover estimate (22)-(23).

Remark 12 The assumptions of Theorem 3 and Theorem 4 are not sharp. However, they allow for simple proofs, and for a wide variety of interaction potentials $W_{1}$ and $W_{2}$. 
Remark 13 Again, as in Remark 2, constraints on the positions of the atoms may be accounted for.

Note that Theorem 4 suggests a strategy for numerically computing the terms of (59). Indeed, it is possible to compute numerically $\psi_{1}$ using its definition (55). Numerical integration then allows to compute $y^{*}$ and the variance $\int_{\mathbb{R}}\left(x-y^{*}\right)^{2} \psi_{1}^{2}(x) d x$. The computation of the infinite sums in (59) is then performed using a simulation of the corresponding Markov chains and taking the expectation value. Note that the law of $Y_{i}$ converges exponentially fast to the invariant measure $\psi_{1}^{2}$ due to the existence of a spectral gap for the transition operator. Hence, the terms $\mathbb{E}\left(Y_{i}-y^{*}\right)$ and $\mathbb{E}\left(\left(\tilde{Y}_{i}-y^{*}\right)\left(\tilde{Y}_{1}-y^{*}\right)\right)$ that appear in the sums in (59) decay exponentially fast, and only a few terms are needed in practice. We will observe in Section 3.1.3 that, on our test example, $A\left(y^{*}\right)$ is already a good approximation of $\langle A\rangle_{N}$. Hence, we have not implemented the strategy just described.

\subsubsection{More than two repatoms}

We explain in this Section how the results of Section 3.1.1 can be adapted to the case when more than two repatoms are considered.

We thus consider the following setting: we have $N+M+1$ atoms of positions $u^{i}$, $0 \leq i \leq M+N$, and $u^{0}, u^{N}$ and $u^{N+M}$ are the repatoms. To remove translation invariance, we set $u^{0}=0$. Since the atoms on the right of $u^{N}$ will not play the same role as those on the left, we denote their distance differently:

$$
y_{i}:=\frac{u^{i}-u^{i-1}}{h} \quad \forall 1 \leq i \leq N, \quad z_{i}:=\frac{u^{i+1+N}-u^{i+N}}{h} \quad \forall 1 \leq i \leq M,
$$

where $h=1 /(N+M)$. We assume that the observable $A$ is a function of $u^{N}-u^{0}$ and $u^{N+M}-u^{N}$. Hence, the expectation value of $A$ reads:

$$
\begin{aligned}
\langle A\rangle_{N, M}= & Z^{-1} \int_{\mathbb{R}^{N+M}} A\left(\frac{1}{N+M} \sum_{i=1}^{N} y_{i}, \frac{1}{N+M} \sum_{i=1}^{M} z_{i}\right) e^{-\frac{\beta}{2} W_{1}\left(y_{1}\right)} \\
& \prod_{i=1}^{N-1} \bar{f}\left(y_{i}, y_{i+1}\right) e^{-\frac{\beta}{2} W_{1}\left(y_{N}\right)} e^{-\beta W_{2}\left(y_{N}+z_{1}\right)} e^{-\frac{\beta}{2} W_{1}\left(z_{1}\right)} \\
& \prod_{i=1}^{M-1} \bar{f}\left(z_{i}, z_{i+1}\right) e^{-\frac{\beta}{2} W_{1}\left(z_{N}\right)} d y d z
\end{aligned}
$$

where, as before, we have set $\bar{f}(x, y)=\exp \left[-\beta W_{2}(x+y)-\frac{\beta}{2} W_{1}(x)-\frac{\beta}{2} W_{1}(y)\right]$. Here again, we may use $\psi_{1}$ defined in (55) in order to rewrite (60) as the expectation value of a function of two independent Markov chains. Indeed, $\psi_{1}$ and $g$ being defined as before (see 
(55) and (57)), we have

$$
\begin{aligned}
\langle A\rangle_{N, M}= & Z^{-1} \int_{\mathbb{R}^{N+M}} A\left(\frac{1}{N+M} \sum_{i=1}^{N} y_{i}, \frac{1}{N+M} \sum_{i=1}^{M} z_{i}\right) \psi_{1}\left(y_{1}\right) e^{-\frac{\beta}{2} W_{1}\left(y_{1}\right)} \\
& \prod_{i=1}^{N-1} g\left(y_{i}, y_{i+1}\right) \frac{e^{-\frac{\beta}{2} W_{1}\left(y_{N}\right)}}{\psi_{1}\left(y_{N}\right)} e^{-\beta W_{2}\left(y_{N}+z_{1}\right)} \psi_{1}\left(z_{1}\right) e^{-\frac{\beta}{2} W_{1}\left(z_{1}\right)} \\
& \prod_{i=1}^{M-1} g\left(z_{i}, z_{i+1}\right) \frac{e^{-\frac{\beta}{2} W_{1}\left(z_{M}\right)}}{\psi_{1}\left(z_{M}\right)} d y d z, \\
= & \mathbb{E}\left[A\left(\frac{1}{N+M} \sum_{i=1}^{N} Y_{i}, \frac{1}{N+M} \sum_{i=1}^{M} Z_{i}\right) \frac{e^{-\frac{\beta}{2} W_{1}\left(Y_{N}\right)-\frac{\beta}{2} W_{1}\left(Z_{M}\right)-\beta W_{2}\left(Y_{N}+Z_{1}\right)}}{\psi_{1}\left(Y_{N}\right) \psi_{1}\left(Z_{M}\right)}\right] \\
& \times\left(\mathbb{E}\left[\frac{e^{-\frac{\beta}{2} W_{1}\left(Y_{N}\right)-\frac{\beta}{2} W_{1}\left(Z_{M}\right)-\beta W_{2}\left(Y_{N}+Z_{1}\right)}}{\psi_{1}\left(Y_{N}\right) \psi_{1}\left(Z_{M}\right)}\right]\right)^{-1},
\end{aligned}
$$

where the sequences $\left(Y_{i}\right)_{i \geq 1}$ and $\left(Z_{i}\right)_{i \geq 1}$ are two independent realizations of a Markov chain of initial law $\psi_{1} e^{-\frac{\beta}{2} W_{1}}$, and of transition kernel $g$. These Markov chains have exactly the same properties as the chain of Section 3.1.1. Hence, we may use again the ergodic theorem as before to prove that:

$$
\frac{1}{N+M} \sum_{i=1}^{N} Y_{i} \longrightarrow L_{1} y^{*}, \quad \frac{1}{N+M} \sum_{i=1}^{M} Z_{i} \longrightarrow L_{2} y^{*}
$$

almost surely, with $L_{1}=N /(N+M)$ and $L_{2}=M /(N+M)$. Thus, the expectation values in (61) simplify, allowing to prove:

Theorem 5 Assume that $A, W_{1}$ and $W_{2}$ satisfy the assumptions of Theorem 3. Assume in addition that $L_{1}=\frac{N}{N+M}$ is fixed, and set $L_{2}=\frac{M}{N+M}=1-L_{1}$. Then, we have

$$
\lim _{N, M \rightarrow+\infty}\langle A\rangle_{N, M}=A\left(L_{1} y^{*}, L_{2} y^{*}\right)
$$

where

$$
y^{*}=\int_{\mathbb{R}} y \psi_{1}^{2}(y) d y .
$$

Here again, it is possible to use an expansion in powers of $1 / N$ and $1 / M$ of the expectation value $\langle A\rangle_{N, M}$. For simplicity, we restrict ourselves to the expansion at first order, and consider the case $N=M$. We assume that the hypotheses of Theorem 4 and Theorem 5 
are satisfied. We then have

$$
\begin{aligned}
\langle A\rangle_{N, N}= & A\left(L_{1} y^{*}, L_{2} y^{*}\right)+\frac{1}{N}\left(\partial_{1} A\left(L_{1} y^{*}, L_{2} y^{*}\right)+\partial_{2} A\left(L_{1} y^{*}, L_{2} y^{*}\right)\right) \sum_{i \geq 1} \mathbb{E}\left(Y_{i}-y^{*}\right) \\
& +\frac{1}{N} \Delta A\left(L_{1} y^{*}, L_{2} y^{*}\right)\left[\frac{1}{2} \int_{\mathbb{R}}\left(x-y^{*}\right)^{2} \psi_{1}^{2}(x) d x\right. \\
& \left.+\sum_{i \geq 2} \mathbb{E}\left(\left(\tilde{Y}_{i}-y^{*}\right)\left(\tilde{Y}_{1}-y^{*}\right)\right)\right]+O\left(\frac{1}{N^{2}}\right)
\end{aligned}
$$

with $L_{1}=L_{2}=1 / 2$. We have not implemented this formula, since, on our test example, $A\left(L_{1} y^{*}, L_{2} y^{*}\right)$ is already a good approximation of $\langle A\rangle_{N, N}$.

\subsubsection{Numerical results}

For the NNN model, we choose the potentials

$$
W_{1}(x)=\frac{1}{2}(x-1)^{4}+\frac{1}{2} x^{2} \quad \text { and } \quad W_{2}(x)=\frac{1}{4}(x-2.1)^{4} .
$$

Note that other choices are possible, such as $W_{1} \equiv W_{2}$, or $W_{2}(x)=W_{1}(x / 2)$ (such that the equilibrium distances of $W_{1}$ and $W_{2}$ are compatible). We have chosen $W_{2}$ such that we observe a significant dependence of ensemble averages (for instance of the mean length $\left\langle u^{N}\right\rangle_{N}$ of the chain) with respect to temperature.

It is important that $W_{1}$ and $W_{2}$ grow fast enough at infinity, such that assumptions of Theorem 3 are satisfied (in particular assumption (58)). As in the NN case, we do not need any convexity assumption on $W_{1}$ and $W_{2}$.

We consider two cases:

- the chain consists of $N+1$ atoms, there are two repatoms $u^{0}=0$ and $u^{N}$, and the observable only depends on the right end atom $u^{N}$. We aim at computing $\left\langle A\left(u^{N}\right)\right\rangle_{N}$. This is the situation of Section 3.1.1.

- the chain consists of $2 N+1$ atoms, there are three repatoms $u^{0}=0, u^{N}$ and $u^{2 N}$, and the observable depends on $u^{N}$ and $u^{2 N}-u^{N}$. We aim at computing $\left\langle A\left(u^{N}, u^{2 N}-\right.\right.$ $\left.\left.u^{N}\right)\right\rangle_{2 N}$. This is a situation covered by Section 3.1.2.

Theorems 3 and 5 respectively provide the asymptotics

$$
\lim _{N \rightarrow+\infty}\left\langle A\left(u^{N}\right)\right\rangle_{N}=A\left(y^{*}\right), \quad \lim _{N \rightarrow+\infty}\left\langle A\left(u^{N}, u^{2 N}-u^{N}\right)\right\rangle_{2 N}=A\left(\frac{1}{2} y^{*}, \frac{1}{2} y^{*}\right) .
$$

We first study the convergence of ensemble averages at the temperature $1 / \beta=1$, as $N$ increases. Results are shown on Figure 12, for a particular choice of observable (we have performed the same tests with other observables, with similar conclusions). We indeed observe that the ensemble averages of the full atom system converge to their Law of Large 
Numbers (LLN) limit, in both cases of two and three repatoms. Note that the exact result for $N=10$ is already very well approximated by the asymptotic limit, $A\left(y^{*}\right)$ in the two repatoms case, $A\left(y^{*} / 2, y^{*} / 2\right)$ in the three repatoms case.
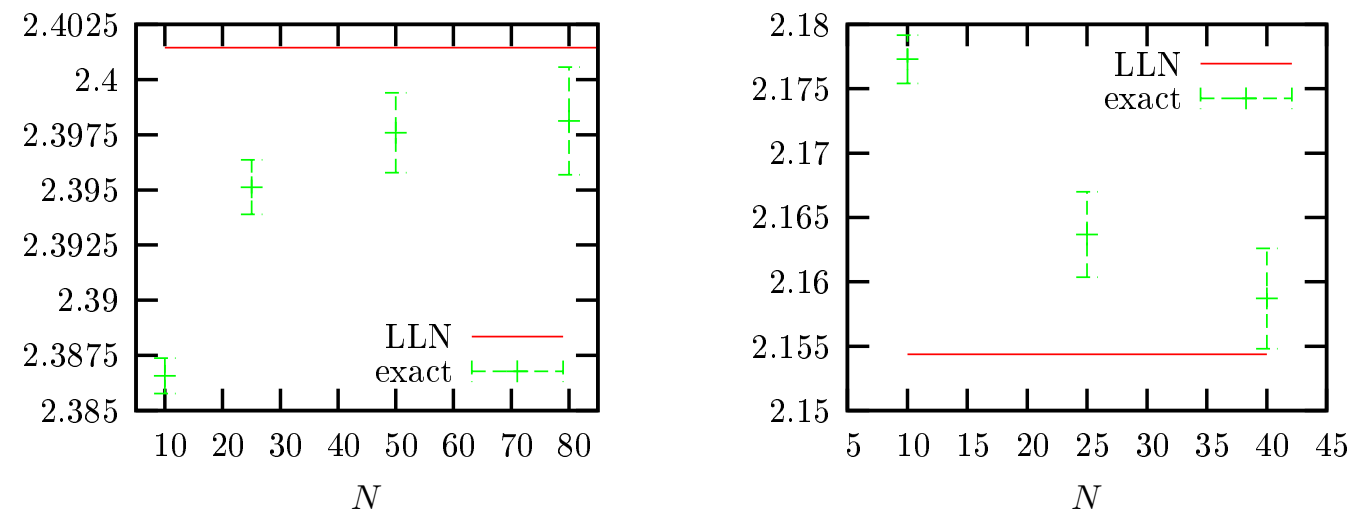

Figure 12: Left graph: convergence, as $N$ increases, of $\left\langle A\left(u^{N}\right)\right\rangle_{N}$ (exact) to $A\left(y^{*}\right)$ (LLN), at the temperature $1 / \beta=1$, for $A(x)=\exp (x)$. Right graph: convergence, as $N$ increases, of $\left\langle A\left(u^{N}, u^{2 N}-u^{N}\right)\right\rangle_{2 N}$ (exact) to $A\left(y^{*} / 2, y^{*} / 2\right)$ (LLN), at the temperature $1 / \beta=1$, for $A(x, y)=\exp (2 x(x+y))$.

We next study the averages as functions of the temperature, for $N=100$. Results are shown on Figure 13. We observe an excellent agreement between the full atom value and the asymptotic limit, in both cases of two and three repatoms, whatever the temperature.

\subsection{Extensions}

In this Section, we briefly explain that our strategy carries out to more general cases.

\subsubsection{The NNNN case}

The case of any finite range interaction may be treated in the same way as we treated the NNN case in Section 3.1.1. Indeed, consider for instance the case of next to next to nearest neighbour interaction (NNNN). In such a case, we are lead to consider (we go back here to 

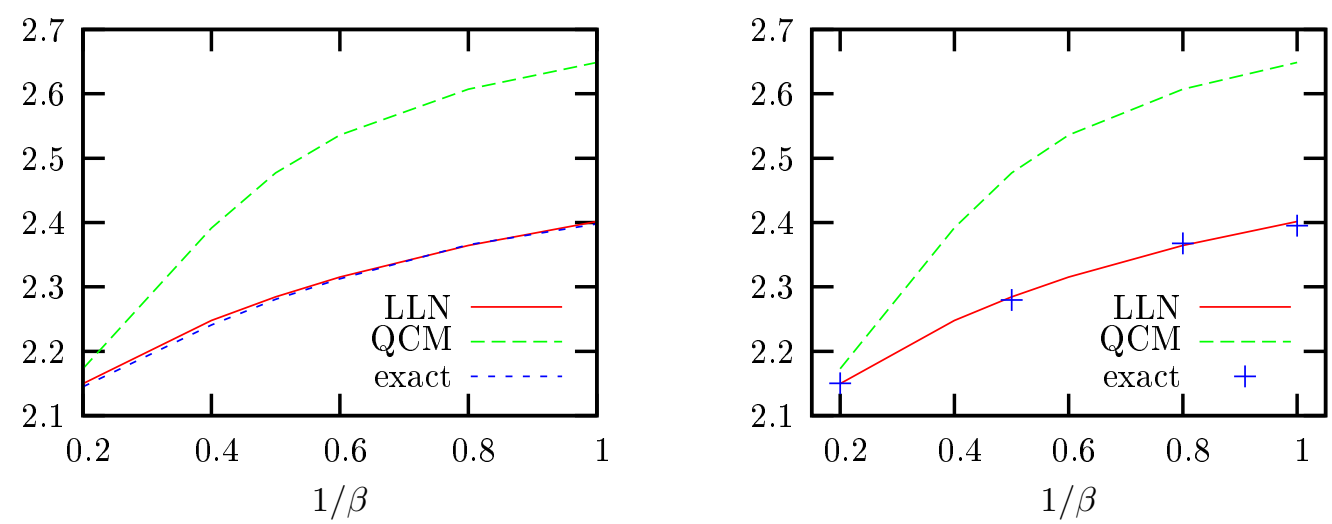

Figure 13: Left graph: we plot $\left\langle A\left(u^{N}\right)\right\rangle_{N},\left\langle A\left(u^{N}\right)\right\rangle_{N}^{\mathrm{QCM}}$ and $A\left(y^{*}\right)$ as functions of the temperature $1 / \beta(N=100, A(x)=\exp (x))$. Right graph: we plot $\left\langle A\left(u^{N}, u^{2 N}-u^{N}\right)\right\rangle_{2 N}$, $\left\langle A\left(u^{N}, u^{2 N}-u^{N}\right)\right\rangle_{2 N}^{\mathrm{QCM}}$ and $A\left(y^{*} / 2, y^{*} / 2\right)$ as functions of the temperature $1 / \beta(N=100$, $A(x, y)=\exp (2 x))$.

the case of 2 repatoms for the sake of clarity):

$$
\begin{aligned}
\langle A\rangle_{N}= & Z^{-1} \int_{\mathbb{R}^{N}} A\left(\frac{1}{N} \sum_{i=1}^{N} y_{i}\right) \exp \left[-\beta \sum_{i=1}^{N} W_{1}\left(y_{i}\right)\right. \\
& \left.-\beta \sum_{i=1}^{N-1} W_{2}\left(y_{i}+y_{i+1}\right)-\beta \sum_{i=1}^{N-2} W_{3}\left(y_{i}+y_{i+1}+y_{i+2}\right)\right] d y_{1} \ldots d y_{N} \\
= & Z^{-1} \int_{\mathbb{R}^{N}} A\left(\frac{1}{N} \sum_{i=1}^{N} y_{i}\right) b\left(y_{N-1}, y_{N}\right) \prod_{i=1}^{N-2} f\left(y_{i}, y_{i+1}, y_{i+2}\right) d y_{1} \ldots d y_{N},
\end{aligned}
$$

where we have set $f(x, y, z)=\exp \left[-\beta W_{1}(x)-\beta W_{2}(x+y)-\beta W_{3}(x+y+z)\right]$, and used the notation $b\left(y_{N-1}, y_{N}\right)=\exp \left[-\beta W_{1}\left(y_{N-1}\right)-\beta W_{1}\left(y_{N}\right)-\beta W_{2}\left(y_{N-1}+y_{N}\right)\right]$ for the boundary term. We assume, for the sake of simplicity, that $N$ is even, i.e.

$$
N=2 M
$$

and define the new variables

$$
\xi_{i}=\left(y_{2 i-1}, y_{2 i}\right), \quad 1 \leq i \leq M
$$


Hence, we have

$$
\begin{aligned}
\langle A\rangle_{N}= & Z^{-1} \int_{\mathbb{R}^{2 M}} A\left(\frac{1}{2 M} \sum_{i=1}^{M} \xi_{i} \cdot(1,1)\right) \sqrt{f\left(y_{1}, y_{2}, y_{3}\right) f\left(y_{2 M-2}, y_{2 M-1}, y_{2 M}\right)} \\
& \times b\left(y_{2 M-1}, y_{2 M}\right) \prod_{i=1}^{M-1} \tilde{f}\left[\xi_{i}, \xi_{i+1}\right] d \xi_{1} \ldots d \xi_{M},
\end{aligned}
$$

where

$$
\tilde{f}[(x, y),(z, t)]=\sqrt{f(x, y, z) f(y, z, t)} .
$$

Hence, this change of variables allows to manipulate again a Markov chain, but in dimension 2. However, the renormalization trick we have used in the NNN case (see Section 3.1.1) cannot be used here, because it relies on the fact that the transition operator is self-adjoint. It is nevertheless possible to use the above structure in the following way: define the operator (on $L^{2}\left(\mathbb{R}^{2}\right)$ )

$$
[P \varphi](z, t)=\int_{\mathbb{R}^{2}} \tilde{f}[(x, y),(z, t)] \varphi(x, y) d x d y,
$$

together with its adjoint

$$
\left[P^{*} \varphi\right](z, t)=\int_{\mathbb{R}^{2}} \tilde{f}[(z, t),(x, y)] \varphi(x, y) d x d y .
$$

Before studying the average (62), let us consider the average

$$
\begin{aligned}
\langle B\rangle=Z^{-1} \int_{\mathbb{R}^{2 M}} B\left(\xi_{i}\right) \sqrt{f\left(y_{1}, y_{2}, y_{3}\right) f\left(y_{2 M-2}, y_{2 M-1}, y_{2 M}\right)} & \\
& \times b\left(y_{2 M-1}, y_{2 M}\right) \prod_{i=1}^{M-1} \tilde{f}\left[\xi_{i}, \xi_{i+1}\right] d \xi_{1} \ldots d \xi_{M}
\end{aligned}
$$

for a continuous and bounded function $B$. Then

$$
\langle B\rangle=Z^{-1} \int_{\mathbb{R}^{2}} B(\xi)\left[P^{i-1} \varphi\right](\xi)\left[\left(P^{*}\right)^{M-i} \psi\right](\xi) d \xi,
$$

where

$$
\begin{array}{r}
\varphi(z, t)=\int_{\mathbb{R}^{2}} \sqrt{f(x, y, z)} \tilde{f}[(x, y),(z, t)] d x d y \\
\psi(t, z)=\int_{\mathbb{R}^{2}} \sqrt{f(z, y, x)} b(y, x) \tilde{f}[(t, z),(y, x)] d x d y .
\end{array}
$$

We assume that the operators $P$ and $P^{*}$ have a simple and isolated largest eigenvalue (which can be proved for many interactions, using for instance Krein-Rutman theorem [40]). Let us denote by $\phi$ and $\phi^{*}$ the corresponding eigenvectors in $L^{2}\left(\mathbb{R}^{2}\right)$, namely

$$
P \phi=\lambda \phi, \quad P^{*} \phi^{*}=\lambda \phi^{*},
$$


where $\lambda=\sup \sigma(P)=\sup \sigma\left(P^{*}\right)$. If $1 \ll i \ll M$, we have the convergence

$$
\langle B\rangle \longrightarrow Z_{\infty}^{-1} \int_{\mathbb{R}^{2}} B(\xi) \phi(\xi) \phi^{*}(\xi) d \xi
$$

where $Z_{\infty}=\int_{\mathbb{R}^{2}} \phi \phi^{*}$. The argument may be adapted to prove that the expectation value $\langle A\rangle_{N}$ defined by $(62)$ converges:

$$
\langle A\rangle_{N} \underset{N \rightarrow+\infty}{\longrightarrow} A\left(y^{*}\right)
$$

where

$$
y^{*}=\frac{\int_{\mathbb{R}^{2}}\left(\xi_{1}+\xi_{2}\right) \phi\left(\xi_{1}, \xi_{2}\right) \phi^{*}\left(\xi_{1}, \xi_{2}\right) d \xi_{1} d \xi_{2}}{\int_{\mathbb{R}^{2}} \phi\left(\xi_{1}, \xi_{2}\right) \phi^{*}\left(\xi_{1}, \xi_{2}\right) d \xi_{1} d \xi_{2}} .
$$

\subsubsection{Polymer chains}

The considerations of Section 2 and Section 3.1 may be easily generalized to the case when the positions $u^{i}$ of the atoms are not restricted to be in the real line, but are vectors of $\mathbb{R}^{2}$ or $\mathbb{R}^{3}$. The only important thing here is that they are indexed by a one dimensional parameter (here, $1 \leq i \leq N)$.

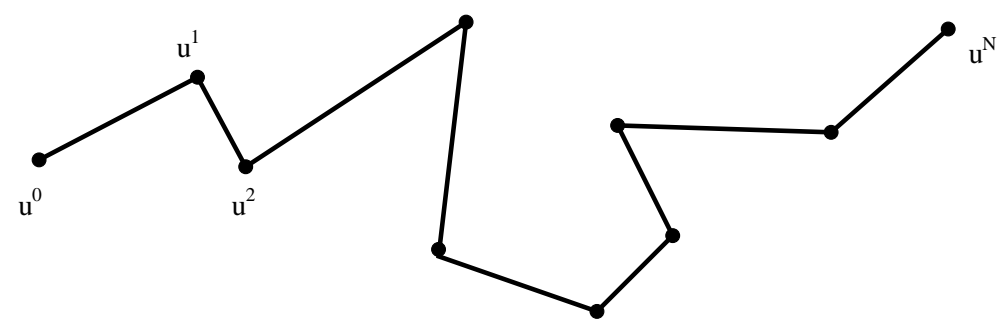

Figure 14: An example of polymer chain. The corresponding model is the same as the present one, except that the positions $u^{i}$ are in $\mathbb{R}^{3}$.

This is the case for instance if one considers a polymer chain (see Figure 14). In such a case, NN or NNN approximations are commonly used, in order to compute the average length of the chain (see [12] for instance). Our approximation strategy carries out to this case.

Acknowledgments: CLB and FL would like to acknowledge stimulating discussions with Mitchell Luskin and Ellad Tadmor, University of Minnesota, Minneapolis. The hospitality 
of the Institute for Mathematics and its Applications (Minneapolis) and of the WeierstrassInstitut für Angewandte Analysis und Stochastik (Berlin) are gratefully acknowledged. Mathias Rousset provided some instrumental input from probability theory. This work has been initiated during the one-year stay (2005-2006) of the fourth author, C.P., at the Ecole Nationale des Ponts et Chaussées, as a visiting graduate student.

\section{References}

[1] M. Anitescu, D. Negrut, P. Zapol, A. El-Azab, A note on the regularity of reduced models obtained by nonlocal quasi-continuum-like approach, Technical Report ANL/MCSP1303-1105, Argonne National Laboratory, Argonne, Illinois, 2005. Available at http://www-unix.mcs.anl.gov/ ^anitescu/PUBLICATIONS/anitescuQC-2007-MP.pdf.

[2] M. Arndt, M. Griebel, Derivation of higher order gradient continuum models from atomistic models for crystalline solids, SIAM J. Multiscale Model. Simul. 4(2), pp 531$562,2005$.

[3] M. Arndt, M. Luskin, Error estimation and atomistic-continuum adaptivity for the quasicontinuum approximation of a Frenkel-Kantorova model, arXiv preprint 0704.1924, 2007.

[4] R. R. Bahadur, R. Ranga Rao, On deviations of the sample mean, Ann. Math. Statist. 31, pp 1015-1027, 1960.

[5] R. J. Baxter, Exactly solved models in statistical mechanics, Academic Press, Inc. London, 1982.

[6] C. M. Bender, S. A. Orszag, Advanced mathematical methods for scientists and engineers, in: International Series in Pure and Applied Mathematics, McGraw-Hill, New York, 1978.

[7] X. Blanc, C. Le Bris, F. Legoll, Analysis of a prototypical multiscale method coupling atomistic and continuum mechanics: the convex case, Acta Math. Appl. Sinica 23(2), pp 209-216, 2007.

[8] X. Blanc, C. Le Bris, F. Legoll, Analysis of a prototypical multiscale method coupling atomistic and continuum mechanics, Math. Mod. Num. Anal. (M2AN) 39(4), pp 797$826,2005$.

[9] X. Blanc, C. Le Bris, P.-L. Lions, From molecular models to continuum mechanics, Arch. Rat. Mech. Anal. 164, pp 341-381, 2002.

[10] X. Blanc, C. Le Bris, P.-L. Lions, Atomistic to Continuum limits for computational materials science, Math. Mod. Num. Anal. (M2AN) 41(2), pp 391-426, 2007.

[11] X. Blanc, F. Legoll, in preparation. 
[12] R. B. Bird, C. F. Curtiss, R. C. Armstrong, O. Hassager, Dynamics of polymeric liquids. Volume 2. Kinetic theory. Second edition, Wiley, New York, 1987.

[13] E. Cancès, F. Legoll, G. Stoltz, Theoretical and Numerical Comparison of Some Sampling Methods for Molecular Dynamics, Math. Mod. Num. Anal. (M2AN) 41(2), pp 351$389,2007$.

[14] S. Curtarolo, G. Ceder, Dynamics of an Inhomogeneously Coarse Grained Multiscale System, Phys. Rev. Lett. 88(25), 255504, 2002.

[15] P. Deák, Th. Frauenheim, M. R. Pederson, Editors, Computer simulation of materials at atomic level, Wiley, 2000.

[16] A. Dembo, O. Zeitouni, Large deviations techniques and applications, Jones and Bartlett Publishers, Boston, MA, 1993.

[17] B. Derrida, Non-equilibrium steady states: fluctuations and large deviations of the density and of the current, J. Stat. Mech. P07023, 2007.

[18] M. Dobson, M. Luskin, Analysis of a force-based quasicontinuum approximation, Math. Mod. Num. Anal. (M2AN) 42(1), pp 113-139, 2008.

[19] L. M. Dupuy, E. B. Tadmor, R. E. Miller, R. Phillips, Finite temperature Quasicontinuum: Molecular dynamics without all the atoms, Phys. Rev. Lett. 95, 060202, 2005.

[20] W. E, Z. Huang, A dynamic atomistic-continuum method for the simulation of crystalline materials, J. Comp. Phys. 182, pp 234-261, 2002.

[21] W. E, P. B. Ming, Cauchy-Born rule and stability of crystals: static problems, Arch. Rat. Mech. Anal. 183(2), pp 241-297, 2007.

[22] W. E, P. B. Ming, Analysis of multiscale methods, J. Comp. Math. 22(2), pp 210-219, 2004.

[23] R. S. Ellis, Entropy, large deviations, and statistical mechanics, volume 271 of Grundlehren der Mathematischen Wissenschaften. Springer-Verlag, New York, 1985.

[24] R. S. Ellis, Large deviations and statistical mechanics. In Particle systems, random media and large deviations, (Brunswick, Maine, 1984), volume 41 of Contemp. Math., pages 101-123. Amer. Math. Soc., Providence, RI, 1985.

[25] R. S. Ellis, An overview of the theory of large deviations and applications to statistical mechanics, Scand. Actuar. J., (1), pp 97-142, 1995. Harald Cram 'er Symposium (Stockholm, 1993).

[26] F. den Hollander, Large deviations. Fields Institute Monographs, 14. American Mathematical Society, Providence, RI, 2000. 
[27] J. Knap, M. Ortiz, An Analysis of the QuasiContinuum Method, J. Mech. Phys. Solids 49(9), pp 1899-1923, 2001.

[28] H. J. Kushner, D. S. Clark, Stochastic approximation methods for constrained and unconstrained systems, volume 26 of Applied Mathematical Sciences. Springer-Verlag, New York, 1978.

[29] R. LeSar, R. Najafabadi, D. J. Srolovitz, Finite-temperature defect properties from freeenergy minimization, Phys. Rev. Lett. 63, pp 624-627, 1989.

[30] P. Lin, Theoretical and numerical analysis of the quasi-continuum approximation of a material particle model, Math. Comput. 72, pp 657-675, 2003.

[31] P. Lin, Convergence analysis of a quasi-continuum approximation for a two-dimensional material, SIAM J. Numer. Anal. 45(1), pp 313-332, 2007.

[32] P.-L. Lions, G. Toscani, A strengthened central limit theorem for smooth densities, J. Funct. Anal. 128, pp 148-176, 1995.

[33] S. P. Meyn, R. L. Tweedie, Markov Chains and Stochastic Stability. Springer (1993).

[34] R. Miller, E. B. Tadmor, R. Phillips, M. Ortiz, Quasicontinuum simulation of fracture at the atomic scale, Modelling Simul. Mater. Sci. Eng. 6, pp 607-638, 1998.

[35] R. Miller, E. B. Tadmor, The Quasicontinuum Method: Overview, applications and current directions, Journal of Computer-Aided Materials Design, 9, pp 203-239, 2002.

[36] S. Olla, Non-equilibrium macroscopic behaviour of chain of interacting oscillators, lecture notes from a course at IHP (Paris), fall 2007.

[37] Ch. Ortner, E. Süli, Analysis of a quasicontinuum method in one dimension, Math. Mod. Num. Anal. (M2AN) 42(1), pp 57-91, 2008.

[38] C. Patz, PhD. dissertation, in preparation.

[39] E. Presutti, Scaling Limits in Statistical Mechanics and Microstructures in Continuum Mechanics, Springer-Verlag, 2008.

[40] H. Schaefer, M. P. Wolff, Topological vector spaces, second edition. Graduate Texts in Mathematics, 3. Springer-Verlag, New York, 1999.

[41] V. B. Shenoy, R. Miller, E. B. Tadmor, R. Phillips, M. Ortiz, Quasicontinuum Models of Interfacial Structure and Deformation, Phys. Rev. Lett. 80(4), pp 742-745, 1998.

[42] V. B. Shenoy, R. Miller, E. B. Tadmor, D. Rodney, R. Phillips, M. Ortiz, An adaptative finite element approach to atomic-scale mechanics - the QuasiContinuum Method, J. Mech. Phys. Solids 47, pp 611-642, 1999. 
[43] E. B. Tadmor, G. S. Smith, N. Bernstein, E. Kaxiras, Mixed finite element and atomistic formulation for complex crystals, Phys. Rev. B 59(1), pp 235-245, 1999.

[44] E. B. Tadmor, R. Phillips, Mixed Atomistic and Continuum Models of Deformation in Solids, Langmuir 12, pp 4529-4534, 1996.

[45] E. B. Tadmor, M. Ortiz, R. Phillips, Quasicontinuum analysis of defects in solids, Phil. Mag. A 73, pp 1529-1563, 1996.

[46] S. R. S. Varadhan, Large deviations and applications, SIAM, Philadelphia, 1984. 


\section{Contents}

1 Introduction $\quad 3$

2 The nearest neighbour (NN) case $\quad 7$

2.1 Limit of the average . . . . . . . . . . . . . . . . . . 9

2.2 Limit of the free energy . . . . . . . . . . . . . . . . . . 12

2.3 Numerical tests . . . . . . . . . . . . . . . . . . 19

3 The NNN case and some extensions $\quad \mathbf{2 4}$

3.1 The next-to-nearest neighbour $(\mathrm{NNN})$ case . . . . . . . . . . . . 25

3.1.1 Limit of the average, the case of two repatoms . . . . . . . . . . 25

3.1 .2 More than two repatoms . . . . . . . . . . . . . . . . . 29

3.1 .3 Numerical results . . . . . . . . . . . . . . . . . . . . . . . 31

3.2 Extensions . . . . . . . . . . . . . . . . . . . . . . . . . 32

3.2 .1 The NNNN case . . . . . . . . . . . . . . . . . 32

3.2 .2 Polymer chains . . . . . . . . . . . . . . . . . 35 
Unité de recherche INRIA Rocquencourt Domaine de Voluceau - Rocquencourt - BP 105 - 78153 Le Chesnay Cedex (France)

Unité de recherche INRIA Futurs : Parc Club Orsay Université - ZAC des Vignes 4, rue Jacques Monod - 91893 ORSAY Cedex (France)

Unité de recherche INRIA Lorraine : LORIA, Technopôle de Nancy-Brabois - Campus scientifique 615, rue du Jardin Botanique - BP 101 - 54602 Villers-lès-Nancy Cedex (France)

Unité de recherche INRIA Rennes : IRISA, Campus universitaire de Beaulieu - 35042 Rennes Cedex (France)

Unité de recherche INRIA Rhône-Alpes : 655, avenue de l'Europe - 38334 Montbonnot Saint-Ismier (France)

Unité de recherche INRIA Sophia Antipolis : 2004, route des Lucioles - BP 93 - 06902 Sophia Antipolis Cedex (France) 